\title{
Parametric generation of second sound in superfluid helium: linear stability and nonlinear dynamics.
}

\author{
Dmitry Rinberg ${ }^{1}$ and Victor Steinberg \\ Department of Physics of Complex Systems \\ The Weizmann Institute of Science, 76100 Rehovot, Israel \\ 1 also: Bell Laboratories, Lucent Technologies, Murray Hill, NJ 07974, USA.
}

(November 3, 2018)

\begin{abstract}
We report the experimental studies of a parametric excitation of a second sound (SS) by a first sound (FS) in a superfluid helium in a resonance cavity. The results on several topics in this system are presented: (i) The linear properties of the instability, namely, the threshold, its temperature and geometrical dependencies, and the spectra of SS just above the onset were measured. They were found to be in a good quantitative agreement with the theory. (ii) It was shown that the mechanism of SS amplitude saturation is due to the nonlinear attenuation of SS via three wave interactions between the SS waves. Strong low frequency amplitude fluctuations of SS above the threshold were observed. The spectra of these fluctuations had a universal shape with exponentially decaying tails. Furthermore, the spectral width grew continuously with the FS amplitude. The role of three and four wave interactions are discussed with respect to the nonlinear SS behavior. The first evidence of Gaussian statistics of the wave amplitudes for the parametrically generated wave ensemble was obtained. (iii) The experiments on simultaneous pumping of the FS and independent SS waves revealed new effects. Below the instability threshold, the SS phase conjugation as a result of three-wave
\end{abstract}


interactions between the FS and SS waves was observed. Above the threshold two new effects were found: a giant amplification of the SS wave intensity and strong resonance oscillations of the SS wave amplitude as a function of the FS amplitude. Qualitative explanations of these effects are suggested.

PACS numbers:43.25.+y, 67.40.Mj, 67.40.Pm

Typeset using REVTEX 


\section{INTRODUCTION.}

Superfluid $\mathrm{He}^{4}$ exhibits rather unique nonlinear acoustic properties particularly in the vicinity of the superfluid transition. As known for a long time [1,2], it is rather easy to observe a second sound shock wave formation even for rather small amplitudes in the close vicinity of the superfluid transition temperature, $T_{\lambda}$ [3, 团. Nonlinear interaction of first sound (FS) and second sound (SS) is another manifestation of nonlinear wave phenomena in the superfluid helium.

There are a few advantages that make the superfluid helium a very appropriate system for quantitative study of nonlinear wave dynamics. First, parameters of nonlinearity can be easily tuned in a wide range by changing temperature in the vicinity of $T_{\lambda}$. Second, thermodynamic and kinetic properties of the superfluid helium are well known. And third, very high precision and accuracy as well as an excellent experimental control, can be achieved in the superfluid helium experiment.

In a contrast to self-interaction of first and second sounds that have been studied a lot [3 5], there were only a few works dedicated to interactions between sounds. The process of SS generation by FS in an infinite geometry was considered theoretically long time ago [6:7]. Two basic mechanisms of FS to SS conversion due to three wave (3W) interaction were predicted: parametric generation of SS and Cherenkov radiation. Here and further, we follow the terminology defined in the pioneer work of Pokrovskii and Khalatnikov [7]. In both cases the finite amplitude SS waves are generated above some threshold amplitude of a FS wave as a result of an instability. In spite of several attempts made during the years [8], an experimental verification of both instabilities was absent. The only experiment dealing with a nonlinear interaction between two sounds was conducted by S. Garrett et al. [9] more than 20 years ago. These authors observed the process of the nonlinear conversion of SS into FS, that was an inverse process to one, considered in Ref. [7]. This observation gave an indication of the existence of the nonlinear interaction between two acoustic branches in the superfluid helium but left aside the problem of the instabilities due to two mechanisms 
predicted theoretically.

A nonlinear interaction of different wave branches is a rather common phenomenon in condensed matter physics. Some of the well known examples are spin waves in ferro- and antiferromagnets interacting with microwaves [10], Langmuir waves in a plasma interacting with electromagnetic waves, two sound branches in a dusty plasma. There are numerous examples of interactions between sound waves and various types of collective oscillations in solids. Some of them are a phonon-plasmon interaction in piezoelectric semiconductors, interaction either longitudinal or shear sounds with electromagnetic waves in piezoelectrics or magnets, an interaction of sound and surface capillary waves. In all these systems, externally driven waves at a sufficiently large amplitude become unstable with respect to generation of waves of another type [10,11]. Only in a very few systems mentioned above, these instabilities were experimentally observed.

This paper is dedicated to one particular example of a wave interaction - a parametric instability. In parametrically driven systems, a driving field excites pairs of waves propagating in almost opposite direction and having approximately a half of a pumping frequency. The threshold of the parametric instability is defined by the balance between an energy, transferred from a driving field to a wave ensemble, and a wave attenuation. At the instability threshold the wave attenuation is compensated by the pumping. Above the instability onset, the wave amplitude should grow exponentially, but saturates due to nonlinear interactions different from one that causes the instability.

The wave dynamics above the onset is defined by nonlinear wave interactions between excited waves. In the most cases amplitudes of parametrically generated waves are sufficiently low, so that only the lowest orders in wave interaction processes, three-wave (3W) and four-wave $(4 \mathrm{~W})$ interactions, should be taken into account. The wave systems can be divided into two major classes according to the type of their dispersion law. The first one is the decay type, which allows $3 \mathrm{~W}$ interaction (for a power type dispersion law, this gives a criteria: $\left.\partial^{2} \omega / \partial k^{2}>0\right)$. In such systems, $3 \mathrm{~W}$ processes are responsible for the amplitude saturation of the parametrically excited waves [11]. These processes provide an energy 
transfer from the resonance frequency to about twice of it, at which the waves attenuate effectively. For the second type of systems with a non-decay type of the dispersion relation $\left(\partial^{2} \omega / \partial k^{2}<0\right), 3 \mathrm{~W}$ processes are forbidden, and $4 \mathrm{~W}$ processes are responsible for the amplitude saturation. The saturation occurs due to "dephasing" of pairs of excited waves in respect to a phase of a pumping frequency [10].

The parametric wave generation is observed in a wide class of nonlinear media. The most prominent examples are spin waves in ferro- and antiferromagnets driven by a microwave field, ferrofluid surface waves subjected to an ac tangential magnetic field, surface waves in liquid dielectrics parametrically excited by an ac electric field, and a parametric excitation of surface waves by a vertical vibration. In the recent years, the latter system, called the Faraday crispation or ripples, was extensively studied due to easy visualization of patterns which appear above the instability threshold [11].

In this paper, we present the first experimental observation of the parametric instability of FS in the superfluid helium. A FS wave with a large enough amplitude is unstable in respect to creation pairs of SS waves propagating almost in opposite directions and having approximately a half of a FS frequency. We study the statistical and dynamic nonlinear properties of the ensemble of the SS waves above the instability threshold, and the interactions of the parametrically generated SS waves with a SS wave pumped externally. The short reports on each of these subjects were published recently elsewhere [12 14 and also presented in Ref. [15.

This paper is written as follows. First, in the Section [1] we present a theoretical background for the wave interactions in the superfluid helium and, particularly, for the parametric instability. The physics related to the threshold phenomena and to behavior of a SS wave ensemble above the threshold is described. At the end of this section, we compare a novel experimental system to study the parametric instability with two well known systems: the Faraday ripples and the spin waves (Sect.[1D). The Section [II is dedicated to an experimental setup. The next Section consists of three major parts. In the Section IVA, the results related to the threshold phenomena: temperature dependence of the threshold, two 
types of a SS spectrum above the threshold and their temperature dependence are presented and discussed. Next, in the Section IVB, we discuss the results related to behavior of the SS wave ensemble above the instability onset. The mechanism of the SS amplitude saturation, the structure of a wave packet above the threshold, its dependence on FS amplitude and temperature, and statistics of the interacting SS waves are considered. The separate Section, (Sect.IVQ), is dedicated to experiments on interaction between an independently generated SS wave with a FS-SS wave ensemble below and above the parametric instability threshold. Here, we present our results on a SS phase conjugation below the onset of instability, a giant SS amplification and strong amplitude fluctuations above the onset. The last section (Sect.V) concludes the paper.

\section{THEORETICAL BACKGROUND.}

\section{A. Wave interaction in superfluid helium}

As was shown in Ref. [7], an effective way to study nonlinear waves is to use a Hamiltonian formalism. The equations of two-fluid hydrodynamics in the Hamiltonian form were first written down in Ref. [16].

Linear properties of waves defined by quadratic terms in Hamiltonian:

$$
H_{2}=\sum_{\mathbf{K}} \Omega_{\mathbf{K}} a_{\mathbf{K}} a_{\mathbf{K}}^{\star}+\sum_{\mathbf{k}} \omega_{\mathbf{k}} b_{\mathbf{k}} b_{\mathbf{k}}^{\star}
$$

and, particularly, by the dispersion relations for FS and SS: $\Omega=c_{1} K$ and $\omega=c_{2} k$, respectively. Here and further, $a$ and $b$ are the wave amplitudes of FS and SS, $c_{1}$ and $c_{2}$ are the FS and SS sound velocities, capital letters $(\Omega, \mathbf{K})$ correspond to the FS frequency and $k$-vectors, and small letters $(\omega, \mathbf{k})$ to SS.

The next term in Hamiltonian, $H_{3}$, is responsible for three wave $(3 \mathrm{~W})$ interactions. This

term is essential only in systems where a dispersion relation allows $3 \mathrm{~W}$ interaction processes. The most general $H_{3}$ term can be written as 


$$
H_{3}=\sum_{\mathbf{k}_{1}, \mathbf{k}_{2}, \mathbf{k}_{3}}\left(V_{\mathbf{k}_{1}, \mathbf{k}_{\mathbf{2}}, \mathbf{k}_{3}} a_{\mathbf{k}_{\mathbf{1}}} a_{\mathbf{k}_{\mathbf{2}}}^{\star} a_{\mathbf{k}_{\mathbf{2}}}^{\star} \delta\left(\mathbf{k}_{\mathbf{1}}-\mathbf{k}_{\mathbf{2}}-\mathbf{k}_{\mathbf{3}}\right)+\text { c.c. }\right),
$$

where $V_{\mathbf{k}_{1}, \mathbf{k}_{2}, \mathbf{k}_{3}}$ is the matrix element of the $3 \mathrm{~W}$ interaction. For particular case of waves in superfluid helium, four different types of $3 \mathrm{~W}$ interaction should be considered. Two of them are self-interactions of FS and SS. The following terms in the Hamiltonian describes these processes: $a a^{\star} a^{\star}$ and $b b^{\star} b^{\star}$ and their complex conjugated terms, corresponding to the inverse processes. Two others are interactions between different branches, FS and SS: $a b^{\star} b^{\star}$ and $a a^{\star} b^{\star}$ and their complex conjugated. These interactions were considered in Ref. [7] and coined as the parametric decay and the Cherenkov emission.

Both sounds have a linear dispersion law that makes interaction very peculiar. The linear dispersion law is a marginal case between the decay type $\left(\partial^{2} \omega / \partial k^{2}>0\right)$ and the nondecay type $\left(\partial^{2} \omega / \partial k^{2}<0\right)$ of spectrum. For the decay type, $3 \mathrm{~W}$ processes are permitted, while for the non-decay type they are forbidden. The linear dispersion law does allow the $3 \mathrm{~W}$ resonant interaction but only for waves propagating along one line that strongly reduces a phase volume of possible interacting waves.

Since the self-interaction of FS is rather weak, the shock waves were observed at rather high incident amplitudes [5]. Contrary to this, in the temperature range near superfluid transition, SS velocity approaches zero, the coefficient of SS self-interaction diverges, and the SS shock wave can be easily generated even at a rather low wave amplitude. This strongly attracted researchers in nonlinear wave dynamics [3, [4, 17].

However, the problem of the nonlinear interactions between many excited SS waves have not been considered. One of the experimental realizations of a multiwave paradigm is an excitation of an ensemble of SS waves by FS via either its parametric decay or Cherenkov emission.

Both of later processes are the decay of one primary wave into two secondary waves. One FS wave in the Cherenkov process generates one FS and one SS waves, and in the parametric decay - two SS waves. In order for such a process to occur, an initial wave should interact with a background noise and amplifies those particular secondary waves 
from the noise, which frequency and $k$-vectors satisfy resonance conditions. The energy transfer from a primary wave is proportional to a primary wave amplitude and a matrix element of interaction, $V a$, and it should exceed the energy attenuated by secondary waves. The threshold amplitude of the decay process is defined by the energy balance between pumping and attenuation. Above the threshold, the mechanisms that are responsible for a secondary wave amplitude saturation, are different from those that generated these waves. These mechanisms need special consideration and will be discussed later for the parametric decay.

Both of these processes have inverse processes of two wave conversion into one wave. Inverse processes do not deal with a background noise wave amplification, so they have no threshold behavior. One of the inverse processes, generation of a double frequency FS wave with a double frequency by two SS waves, was experimentally studied by S. Garrett et al. [9].

\section{Cherenkov radiation of SS.}

The Cherenkov emission results from the resonance interaction between a FS wave with the frequency $\Omega$ and the wavevector $\mathbf{K}$ with a pair of waves: a SS wave $\left(\omega^{\prime}, \mathbf{k}^{\prime}\right)$ and a FS wave $\left(\Omega^{\prime}, \mathbf{K}^{\prime}\right)$, satisfying the following resonance conditions (see vector diagram in Fig.1.a ) :

$$
\Omega=\omega^{\prime}+\Omega^{\prime}, \quad \mathbf{K}=\mathbf{k}^{\prime}+\mathbf{K}^{\prime} .
$$

Here $\Omega=c_{1} K, \omega=c_{2} k$ are related to the FS and SS waves, respectively. Due to large difference in FS and SS velocities, particularly near the superfluid transition, $\eta=c_{2} / c_{1} \ll 1$, the generated SS wave propagates almost in the forward direction, having the frequency $\omega^{\prime}=2 \Omega \eta \sin (\chi / 2)$, and the secondary FS wave propagates in the backward direction with almost the same frequency and the wavenumber, as the incident FS wave.

The strength of the Cherenkov interaction which is defined by the matrix element 
$V(\Omega, \chi)$, was calculated in Ref. [7]. The FS threshold amplitude for the Cherenkov radiation is defined as

$$
a_{t h}^{\infty}=\frac{\sqrt{\gamma_{1}\left(\Omega^{\prime}\right) \gamma_{2}\left(\omega^{\prime}\right)}}{V(\Omega, \chi)} .
$$

It has minimum at $\chi=\pi$, and is scaled with the frequency as $a_{t h}^{\infty} \sim \sqrt{\Omega}$. Here $\gamma_{1}$ and $\gamma_{2}$ are the attenuation rates of the FS and SS waves, respectively.

\section{Parametric decay instability.}

Another 3W resonance process between the FS and SS waves is the parametric decay of one FS wave into two SS waves:

$$
\Omega=\omega_{1}+\omega_{2}, \quad \mathbf{K}=\mathbf{k}_{\mathbf{1}}+\mathbf{k}_{\mathbf{2}}
$$

Due to smallness of the ratio $\eta$, the parametrically generated SS waves have almost a half the FS frequency, $\omega_{1,2} \approx \Omega / 2$, and propagate in almost opposite directions, $\mathbf{k}_{\mathbf{1}} \approx-\mathbf{k}_{\mathbf{2}}$. The vector diagram of this process is shown in Fig.1. 1.b. A more detailed analysis of the resonance conditions reveals an angular dependence of the SS frequency

$$
\omega_{1,2}=\frac{\Omega}{2}(1 \pm \eta \cos \theta)
$$

where $\theta$ is the angle between the plane of the SS wave propagation direction and the FS wave vector, see Fig.11.b. In $k$-space, the resonance surface of SS waves interacting with the FS wave, is the ellipsoid of revolution (almost a sphere). The matrix element of the parametric decay process has been also derived in Ref. [7] and has the following form

$$
U(\Omega, \theta)=\sqrt{\frac{\Omega^{3}}{32 \rho}} c_{1}^{-1}\left(U_{1}+U_{2}-2 \cos ^{2} \theta\right),
$$

where $\rho$ is the density of a liquid helium, and $U_{1}$ and $U_{2}$ are the thermodynamic functions of temperature, pressure, density, and superfluid density:

$$
\begin{aligned}
U_{1} & =-\frac{\rho}{J \phi}\left(\frac{C_{P}}{T} \frac{\partial \psi}{\partial P}+\frac{\alpha_{P}}{\rho} \frac{\partial \phi}{\partial T}\right), \\
U_{2} & =\rho^{-1}\left(\frac{\partial \psi}{\partial T}+\frac{\alpha_{P}}{\kappa_{T}} \frac{\partial \psi}{\partial P}\right) .
\end{aligned}
$$


Here the following notations are introduced: $\psi=\rho \alpha_{P} / J, J=\rho \kappa_{T} C_{P} / T-\alpha_{P}^{2}, \sigma=S / \rho$, $\phi=\rho_{s} / \rho$, where $\rho$ is the helium density, $\rho_{s}$ is the superfluid density, $\alpha_{P}=-\rho^{-1}(\partial \rho / \partial T)_{P}$ is the isobaric thermal expansion, $\kappa_{T}=\rho^{-1}(\partial \rho / \partial p)_{T}$ is the isothermal compressibility, $C_{P}=$ $T(\partial \sigma / \partial T)_{P}$ is the specific heat, $S$ is the entropy per unit mass.

A FS wave of an amplitude $a$ generates SS waves at the rate $|a U|$. The latter dissipate at the rate $\gamma_{2}$. The parametric instability occurs when the SS amplification exceeds the dissipation. Then the FS threshold amplitude in an infinite geometry is defined as

$$
a_{t h}^{\infty}=\frac{\gamma_{2}(\Omega / 2)}{U(\Omega, \theta)},
$$

The function $a_{t h}^{\infty}(\theta)$ has a very shallow minimum for a symmetric decay at $\theta=\pi / 2$, when two SS waves have exactly a half the FS pumping frequency.

\section{Experimental conditions for observation of both instabilities.}

According to the estimates made in Ref. [7], the threshold intensities of the FS for both processes differ substantially. Frequencies of the SS waves excited in these processes also differ greatly. In the Cherenkov emission, the frequency of the excited SS waves is of the order of $2 \Omega\left(c_{2} / c_{1}\right)$, and in the parametric decay the SS frequency is equal to $\sim \Omega / 2$. As a result, it was found in Ref. [7], that in an infinite system the temperature range, where the parametric excitation precedes the Cherenkov emission, is located between 0.9 and $1.2 \mathrm{~K}$. However, as it will be shown later, the corresponding dissipation length for the SS waves is too large in this region, and any experimental cell cannot be considered as an infinite one. The finite size effect drastically alters the threshold value of the FS amplitude and makes impossible the observation of the instabilities. So, from this point of view, the vicinity of the superfluid transition is only possible temperature range where the effect can be observed.

Our estimates that took into account both regular and singular parts of the thermodynamic and kinetic properties of the superfluid helium near $T_{\lambda}$ [18] showed that the threshold value of the parametric instability was almost independent of $\tau$ in the range of the reduced 
temperatures from $10^{-6}$ to $10^{-2}$. For the Cherenkov instability the threshold had depen-

dence $\tau^{-0.67}$ in the same temperature range and was much higher then that of the parametric instability. Thus, the parametric instability undoubtfully precedes the Cherenkov radiation in the vicinity of $T_{\lambda}$.

The advantage to work in the vicinity of $\lambda$-point manifests in a possibility to deal with the SS waves with the attenuation length, $l$, that is less or comparable to the cell size. The attenuation length of the SS varies drastically in this temperature range [19]. This makes consideration of finite size effects very important.

\section{B. Parametric Instability: threshold related phenomena}

From here, the rest of the paper will be dedicated to the parametric excitation of the SS waves. In order to create a large enough amplitude of FS excitation, the resonant cavity cell was built (see Sect.IIA). SS propagates mostly in the direction perpendicular to a FS $k$-vector, along the planes of FS transducers. So, two effects due to a cell size and boundary conditions should be considered. One is a finite cell size effect in the direction of SS propagation, another is a resonant effect due to reflections from the cavity walls in the perpendicular direction.

\section{Finite size effect.}

Since a horizontal size of experimental cells used in our experiments, is comparable with the attenuation length of the SS waves, the lateral boundary conditions should be taken into account to define the FS threshold amplitude for the parametric instability. A straightforward generalization of the envelope method 20,21] for a system of a size $L$, where waves arrive and reflect normally to the boundaries, with an arbitrary reflection coefficient $r$, yields the following expression for the threshold [12]:

$$
a_{t h}=a_{t h}^{\infty}\left[1+\xi^{2}(l / L)^{2}\right]^{1 / 2}
$$


where $\xi$ is the minimal positive root of the equation

$$
\tan \xi=-\frac{\left(1-r^{2}\right) \xi(l / L)}{1+r^{2}-2 r\left[1+\xi^{2}(l / L)^{2}\right]^{1 / 2}} .
$$

Here $a_{t h}^{\infty}$ is the threshold value for an infinite cell. For $L<l$, the value of $a_{t h}$ (along its temperature dependence) is mainly determined by the second term in the parenthesis in Eq.(11), i.e., $a_{t h} \sim c_{2} /(L U) \propto \tau^{1.33}$ (see curves $\mathbf{B}$ in Fig.8). Note that if the reflection coefficient tends to unity, the threshold coincides with that for an infinite system: $r=1$, $\xi=0, a_{t h}=a_{t h}^{\infty}$. For zero reflection coefficient and large ratio $l / L, \xi$ tends to $\pi / 2$, and one obtains $a_{t h} \approx a_{t h}^{\infty} \cdot \pi / 2 \cdot l / L$.

\section{Resonance cavity effects.}

As we already mentioned, in order to increase the attainable value of the FS amplitude in the cell, the resonance cavity for the FS has been used. Thus, the SS waves are generated by the FS standing wave. One can consider two parametric excitation processes caused by two components of the FS standing wave, with the wave vectors $\pm \mathbf{K}$ (Fig.21). The momentum conservation conditions for these processes are

$$
\mathbf{K}=\mathbf{k}_{\mathbf{1}}+\mathbf{k}_{\mathbf{2}}, \quad-\mathbf{K}=\mathbf{k}_{\mathbf{3}}+\mathbf{k}_{\mathbf{4}} .
$$

The processes do not interfere unless the same SS phonon participates in the both of them. If it occurs, e.g., at $\mathbf{k}_{\mathbf{1}} \equiv \mathbf{k}_{\mathbf{3}}$, the both components of the FS standing wave contribute to

the excitation process. Therefore this process has a lower threshold [22]: $a_{t h}=a_{t h}^{\infty} / \sqrt{2}$. The frequencies of the generated SS waves in this case are

$$
\omega=\frac{\Omega}{2}\left(1 \pm \eta^{2}\right)
$$

These both frequencies can be observed only if the frequency splitting between two SS waves, $\omega_{1}-\omega_{2}=\Omega \eta^{2}$, is larger than the SS attenuation, i.e., $\Omega \eta^{2}>\gamma_{2}$. The SS waves with wave vectors $\mathbf{k}_{\mathbf{2}}$ and $\mathbf{k}_{\mathbf{4}}$ interact with different components of the FS standing wave, $+\mathbf{K}$ and $-\mathbf{K}$. In the opposite case, $\Omega \eta^{2}<\gamma_{2}$, i.e., the frequency splitting is less than the SS 
attenuation, each SS wave interacts with both FS waves. The frequencies of all SS waves must be equal : $\omega_{1,2}=\Omega / 2$. This also reduces the threshold amplitude of FS by a factor of two, compared with that in an infinite cell [22]:

$$
a_{t h}=a_{t h}^{\infty} / 2
$$

In general, the threshold of the parametric excitation of the SS waves by the FS standing wave in a finite horizontal size resonance cavity for FS waves, can be written in the following way $12,15,22$ :

$$
a_{t h}=a_{t h}^{\infty} \zeta\left(\frac{\Omega \eta^{2}}{\gamma_{2}}\right) \sqrt{1+\left[\xi\left(r, \frac{l}{L}\right) \frac{l}{L}\right]^{2}},
$$

where $\zeta$ is the numerical function of the ratio $\Omega \eta^{2} / \gamma_{2}$ with limits:

$$
\begin{array}{ll}
\Omega \eta^{2} / \gamma_{2} \ll 1, & \zeta=1 / 2, \\
\Omega \eta^{2} / \gamma_{2} \gg 1, & \zeta=1 / \sqrt{2} .
\end{array}
$$

\section{Splitting of SS waves spectra.}

The resonant cavity effect leads to the discreteness of the SS wave vector in the direction of a FS wave propagation and to another interesting phenomenon, namely, the existence of two types of spectra of the SS waves and the transition between them as a function of closeness to $T_{\lambda}$. Far away from $T_{\lambda}$, two equidistant peaks around $\Omega / 2$ should be observed, while closer to $T_{\lambda}$, a single sharp peak at exactly $\Omega / 2$ frequency should appear. Indeed, as we already mentioned in the previous subsection, the frequency splitting between two SS waves, generated by two components of the FS standing waves in the resonance cavity, should be compared with the SS attenuation. Far away from $T_{\lambda}$, one has $\Omega \eta^{2}>\gamma_{2}$, and two discrete lines in the spectra are present. In the opposite case, $\Omega \eta^{2}<\gamma_{2}$, when the SS attenuation is larger than the spectrum splitting, just one line at exactly $\Omega / 2$ is found. Thus the discreteness of the resonance states is smeared out closer to $T_{\lambda}$. From the equality $\Omega \eta^{2}=\gamma_{2}$ the transition temperature from one-peak to two peaks spectra is defined. 


\section{Properties of SS waves above the threshold}

\section{Nonlinear amplitude saturation mechanism.}

Recently A. Muratov [22] has published a theory, based on the Hamiltonian approach, which describes nonlinear properties of an ensemble of weakly interacting SS waves above the parametric instability threshold. Let us review these results. The crucial point in the understanding the nonlinear behavior of the weakly interacting SS waves, is the nature of a nonlinear saturation mechanism of the instability. First, it was shown [22], that for the SS waves where $3 \mathrm{~W}$ interaction is permitted, the nonlinear attenuation is significantly more important than the renormalization of a pumping field. The latter mechanism was thoroughly studied in the spin wave systems [10]. In the case of the SS waves, the $3 \mathrm{~W}$ interaction provides a very effective channel to dissipate the energy of the parametrically excited waves by the second harmonic generation, i.e. generation of the SS waves with $2 k$ wave vector and $2 \omega$ frequency. It becomes essential only if an angle between the wave vectors

of the interacting SS waves is smaller than $4 \sqrt{\gamma_{2} / \omega}$. Indeed, the $3 \mathrm{~W}$ interaction couples a linearly unstable mode $(k, \omega)$, which is amplified by the FS pumping, with a linearly stable mode $(2 k, 2 \omega)$, which is dissipative.

The theory predicts the functional relation between the total density (or intensity), $I_{2}$, of the parametrically excited waves and the reduced pumping amplitude or the control parameter $\epsilon=a / a_{t h}-1$. For the mechanism of the nonlinear attenuation considered in the Muratov's work [22], the functional dependence is linear:

$$
I_{2}=g \epsilon,
$$

where, $g$ is the coefficient defined by the dimensionality and the shape of the phase volume of the excited waves which is determined by the symmetry of the system (boundary conditions), by the phase volume of the pumping waves, and by the properties of the interaction vertex between the pumping wave and the parametrically generated waves. $I_{2}$ is maximal for $3 \mathrm{D}$ wave excitation, i.e., generation of waves on a spherical $k$-surface, and minimal for 1D wave 
excitation, i.e., generation of a flat wave. Waves with a linear dispersion law interact through $3 \mathrm{~W}$ process only if they propagate in almost same direction. So, for a higher dimensionality of a phase volume of excited waves, the density of waves propagating in the same direction is lower. In a contrary, in one dimensional wave excitation case, all waves propagates along the same direction. So, for the higher dimensionality the energy transfer to the double frequency should be weaker, and the intensity of the parametrically excited waves is higher.

The proportionality of the SS intensity, $I_{2}$, to the control parameter, $\epsilon$, is the distinguish property of the nonlinear attenuation mechanism of the parametric wave saturation. The "dephasing" mechanism leads to the different relation: $I_{2} \sim \epsilon^{1 / 2} \| 10$.

The coefficient $g$ was calculated in Ref. [22] for the cases with different dimensionality. As we will show further, the dimensionality of the excited SS waves in two experimental cells differs due to different lateral boundary conditions. In the cell I with the reflecting boundaries the SS waves were one-dimensional, or almost flat. While in the cell II without reflection the SS waves were, probably, two-dimensional. The difference in the intensity of $2 \mathrm{D}$ and $1 \mathrm{D}$ waves is a factor $\sqrt{\gamma_{0} / \omega_{0}}$, that is rather small [22]. Since the experimentally observed intensity of the excited SS waves is much smaller than the theoretically obtained value even for $1 \mathrm{D}$ case, we use the latter case for comparison. In this case the coefficient $g$ in the expression for the total intensity of the parametrically excited SS waves (see Eq.(17)) is

$$
g=c_{2} \omega\left(\frac{\gamma_{2}}{2 B}\right)^{2}
$$

where $B \equiv B(\theta=0)$ is the triple vertex of the SS interaction. The latter at the arbitrary angle $\theta$ between the SS wave vectors in $P$ and $T$ variables looks as [22]:

$$
B(\theta)=\sqrt{\frac{\omega^{3} \chi}{16 \rho}}\left\{\chi^{-1} \mathbf{D}(\chi)+\phi^{-1} \mathbf{D}(\phi)[\cos (2 \theta)-2 \cos \theta]\right\},
$$

where $\chi=\rho \kappa_{T} / J, \mathbf{D}=\frac{\partial}{\partial T}+\frac{\alpha_{P}}{\kappa_{T}} \frac{\partial}{\partial P}$, and $\psi$ and $J$ are defined after Eqs. (8) and (9). In order to estimate the value of the intensity of the excited SS waves at a given value of the control parameter of the instability, $\epsilon$, one needs to evaluate the triple vertex of the SS waves 
interaction. By simple algebra the expression for $B(\theta=0)$ can be rewritten in the following form:

$$
\begin{aligned}
B(0) & =B_{1}+B_{2} \\
B_{1} & =c_{1} \rho U \sqrt{\frac{2}{\psi}\left(\frac{\omega}{\Omega}\right)^{3}\left(\frac{\partial T}{\partial P}\right)_{\rho}} \\
B_{2} & =\sqrt{\frac{\omega^{3}}{16 \rho \psi}\left(\frac{\partial T}{\partial P}\right)_{\rho} \frac{\rho \alpha_{P}}{J \phi} \frac{\partial \phi}{\partial P}\left[\left(\frac{\partial T}{\partial P}\right)_{S}^{-1}-\left(\frac{\partial T}{\partial P}\right)_{\rho}^{-1}\right] .}
\end{aligned}
$$

Let's estimate the triple vertex $U$ of the FS and SS wave interaction at the reduced temperature $\tau=3.3 \times 10^{-4}$ using the expression for the threshold intensity of FS:

$$
I_{1}=\left(2 \rho c_{1}\right)^{-1}(\delta P)^{2}=c_{1} \Omega\left(\frac{\gamma_{2}}{2 U}\right)^{2}
$$

At these conditions one finds $\gamma_{2}=84(\mathrm{sec})^{-1}, c_{1}=2.177 \times 10^{4}(\mathrm{~cm} / \mathrm{sec}), C_{P}=56.2(\mathrm{~J} / \mathrm{molK})$, $\alpha_{P}=-0.065\left(K^{-1}\right)$, and the experimental value of the threshold pressure amplitude of the FS wave is $\delta P=50 P a$. Then one gets $U=3.5 \times 10^{5}(\mathrm{~cm} / \mathrm{g} \cdot \mathrm{sec})^{1 / 2}$, and for $B_{1}=10^{6}(\mathrm{~cm} / \mathrm{g}$. $\mathrm{sec})^{1 / 2}$ and $B_{2}=3.8 \times 10^{4}(\mathrm{~cm} / \mathrm{g} \cdot \mathrm{sec})^{1 / 2}$. Here we estimate $\kappa_{T}=1.49 \times 10^{-8}\left(\mathrm{~cm}^{2} / \mathrm{dyn}\right)$, $J=0.136\left(K^{-2}\right), \psi=-0.07\left(\mathrm{~g} \cdot \mathrm{K} / \mathrm{cm}^{3}\right),\left(\frac{\partial T}{\partial P}\right)_{\rho}=-2.29 \times 10^{-7}\left(\mathrm{~K} \cdot \mathrm{cm}^{2} / \mathrm{dyn}\right),\left(\frac{\partial T}{\partial P}\right)_{S}=$ $-6.9 \times 10^{-9}\left(\mathrm{Kcm}^{2} / \mathrm{dyn}\right)$, and $\frac{\partial \ln \left(\rho_{S} / \rho\right)}{\partial P}=-8.3 \times 10^{-6}\left(\mathrm{~cm}^{2} / \mathrm{dyn}\right)$. Thus, finally one obtains the following value for the coefficient:

$$
g=4.5 \times 10^{-3}\left(\mathrm{erg} / \mathrm{cm}^{2} \mathrm{sec}\right)=4.5 \times 10^{-4}\left(\mu \mathrm{W} / \mathrm{cm}^{2}\right)=4.5 \times 10^{-6}\left(\mathrm{~W} / \mathrm{m}^{2}\right)
$$

The latter one should be compared at, e.g., $\epsilon=1$ with $I_{1}=0.04\left(\mathrm{~W} / \mathrm{m}^{2}\right)$ at the same conditions.

As we have already pointed out the nonlinear attenuation is proportional to the strength of the interaction between the SS waves. The same type of interaction is responsible for the creation of the SS shock waves which strength increases strongly while approaching the superfluid transition. Thus one can expect that the nonlinear attenuation can increase as well near $T_{\lambda}$. Then the intensity of the SS waves parametrically generated above the threshold, should decrease correspondingly with the scaling $g \sim \tau^{\alpha}$, where $\alpha>0$. Indeed, the estimated scaling of asymptotic temperature behavior of the coefficient $g$ is $g \sim \tau^{0.33}$. 


\section{Broadening of the SS spectrum above the threshold.}

Another manifestation of the nonlinear wave interaction is a spectral broadening of the parametrically generated waves. In weakly dissipative nonlinear media, $3 \mathrm{~W}$ and $4 \mathrm{~W}$ play different roles in wave kinetics. Although $3 \mathrm{~W}$ and $4 \mathrm{~W}$ processes control the amplitude saturation of the parametrically excited waves for the decay and nondecay types of the dispersion law respectively, only the $4 \mathrm{~W}$ processes allow the wave interactions within a narrow frequency range around the main peak at the parametric resonance frequency. Therefore, the spectral shape around the peak is solely controlled by the $4 \mathrm{~W}$ processes. Thus, one can expect that the spectral shape at the parametric resonance frequency exhibits some universal properties independent of details of the wave interaction and the type of the dispersion law. Wave amplitudes in different parametrically driven systems can be limited by different nonlinear mechanisms, their spectral broadening mechanism is universal.

The first experimental evidence and following up theoretical explanation of the exponential spectra based on a kinetic theory, were obtained for the parametrically generated spin waves 23,24]. A theoretical idea behind the explanation is very general and convincing one [23]. There are two reasons for the spectral broadening: a thermal noise above the onset and a "intrinsic" noise due to 4W scattering of spin-waves. The latter becomes important at higher values of the control parameter.

The broadening due to the thermal noise produces the squared Lorentzian shape spectrum [10]. The "intrinsic" noise results from the $4 \mathrm{~W}$ interaction. This interaction does not transfer energy far away from the region of the parametric resonance frequency peak, but pumps it to neighboring modes and broadens the spectrum. In general, the wave packet shape is described by a nonlinear integral equation for wave amplitude correlation functions. It was proved that such equation has universal exponentially decaying tails in the frequency domain 25]. The $4 \mathrm{~W}$ resonance interaction is present in any medium with parametrically

driven waves. Thus, the kinetic theory predicts the universal spectral broadening in such systems irrespectively of the wave dispersion law. 
A theory of the spectral broadening in the parametrically excited SS waves was developed recently by A. Muratov [22]. According to Ref. [22], the thermal width of the central line in the SS spectrum can be estimated from the following expression

$$
\frac{(\Delta \omega)_{T}}{\gamma_{2}}=\frac{\omega^{2} \gamma_{2} k_{B} T}{4 \pi^{2} c_{2}^{2} I_{2}},
$$

where $k_{B}$ is the Boltzmann constant, and $I_{2}=g \epsilon$ is the SS waves intensity, Eq.(17). Using estimates for $g$ from the previous section and from Ref. [22], at $\epsilon=1$ one obtains $(\Delta \omega)_{T} / \gamma_{2} \sim$ $10^{-6}$, which is extremely small and unattainable in a real experiment.

There are two possible channels for the "intrinsic" noise. The first is a direct $4 \mathrm{~W}$ process, i.e. direct scattering of two SS waves on two SS waves. The second is two consequent $3 \mathrm{~W}$ processes: Two SS waves generate a double frequency SS wave which decays into two new SS waves with a frequency of the initial waves. This scattering process can be considered also as an indirect $4 \mathrm{~W}$ process. As calculations show [22], such second order $3 \mathrm{~W}$ scattering (or the indirect $4 \mathrm{~W}$ process) is more effective than the direct $4 \mathrm{~W}$ scattering of two $\mathrm{SS}$ waves on two SS waves. The "intrinsic" reduced width of the central line in the SS spectrum depends on the dimensionality of the excited SS waves [22]

$$
\frac{\Delta}{\gamma_{2}} \sim \epsilon^{8 /(9-D)} \cdot(1+\epsilon)^{\frac{D-1}{9-D}}
$$

where $D$ is the space dimensionality of the SS waves. Thus, at $D=2$ one has $\Delta / \gamma_{2} \sim$ $\epsilon^{8 / 7} \cdot(1+\epsilon)^{1 / 7}$, and at $D=1$ one gets $\Delta / \gamma_{2} \sim \epsilon$.

\section{Comparison of different parametrically driven wave systems.}

At this point, it will be instructive to compare the parametric instability in the novel system with another two systems which were studied the most extensively: the spin waves in magnetics generated by a microwave pumping, and the Faraday ripples excited on a fluid free surface by vertical vibrations. From a theoretical point of view, these two systems

exhibit different aspects of a nonlinear behavior of the parametrically excited waves. The 
Faraday crispation is considered as a canonical example of a pattern forming system, and was studied by using an amplitude equation dynamical approach [11. Whereas the spin waves exhibit very large number of interacting modes. In this system, an irregular state of excited waves is expected to appear above the threshold of the parametric instability [10]. Then the statistical kinetic approach, based on a random phase approximation, is used to describe experimental results [10]. The main distinction of the parametrically excited spin waves is an enormous aspect ratio, $\Gamma=L / l$, which is usually 100 and more (at $L k \approx 10^{5}-10^{6}$ ) at very minute relative dissipation rate, $\gamma / \omega \leq 10^{-6}$. Here $L$ is the horizontal cell size, $l$ and $\gamma$ are the attenuation length and the dissipation rate of the waves, respectively. The dispersion law of the spin waves in most systems is of the non-decay type. In this case, the $3 \mathrm{~W}$ resonant interactions of the spin waves above the threshold are forbidden, and only the $4 \mathrm{~W}$ resonant interactions are possible. As was suggested theoretically and verified experimentally, a non-dissipative mechanism of the $4 \mathrm{~W}$ interactions is responsible for a saturation of spin waves amplitude [10]. This mechanism involves a phase detuning ("dephasing") between the spin waves and the electromagnetic rf pumping. Even though, the $4 \mathrm{~W}$ interactions is of the next order approximation in the wave amplitude compared to the $3 \mathrm{~W}$ case, the $4 \mathrm{~W}$ interactions are responsible for the amplitude saturation, when $3 \mathrm{~W}$ processes are forbidden by the dispersion relation [11]. Due to all these features the wave turbulent-like state is observed just above the instability threshold.

At this point we would like to clarify various definitions of highly irregular wave states. The term "wave," or "weak," turbulence was introduced to distinguish the highly irregular state of many interacting waves from a strong hydrodynamic turbulence [10,26]. A common feature of a fully developed wave and a hydrodynamic turbulence is an observation of a wide spectrum of excited modes in the frequency and wavenumber domains, where the energy pumping and the dissipation have very different scales. The major factor that makes wave (weak) turbulence different from the hydrodynamic one is a presence of a small parameter in a theory, such as, e.g., ratio of a wave energy to a ground state energy. While there is no theory of hydrodynamic turbulence, the self consistent perturbation theory of wave 
turbulence has been developed in the works of Zakharov's school [26]. One of the strong assumptions of this theory is the randomness of the phases of waves creating turbulent state [10].

The word "turbulence" in a context of wave problems sometimes has different meaning. The irregular wave state with many interacting waves can be also called turbulence. In the case of parametric pumping, such a state has been coined as a parametric turbulence [10]. In this case, in a contrast to the developed wave turbulence, the energy pumping and the dissipation occurs on the same spatial scale. A narrow, almost singular spectrum characterizes the parametric turbulence. The theory of the parametric turbulence was first developed and applied to a spin wave system [10]. Thus, the parametric wave turbulence is the irregular state of waves with random phases, that consists one or several wave packets and does not exhibit an inertial range with a wide range of algebraic power law in a spectrum, like in the fully developed wave turbulence [10,26].

The parametrically generated surface waves exhibit a very different type of behavior depending on the value of $k$-vector. In a small $k$ limit, gravity waves with thenon-decay type spectrum $\left(\partial^{2} \omega / \partial k^{2}<0\right)$ are excited, while in a large $k$ limit, capillary waves with the decay type spectrum $\left(\partial^{2} \omega / \partial k^{2}>0\right)$ are found In the intermediate regime of the capillary-gravity waves, there exists the value of $k$ at which only collinear resonant $3 \mathrm{~W}$ interactions are permitted. It is coined as the second-harmonic resonance, and the corresponding capillary-gravity waves are called the Wilton's ripples [27].

In the capillary wave regime, In the capillary wave regime, $3 \mathrm{~W}$ resonance conditions define the angle of strong nonlinear interactions. These interactions are responsible for both amplitude saturation and pattern formation [28,29,?]. In a sufficiently large container, $L k \gg 1$, the standing surface waves above the threshold produce patterns which symmetry is found to be independent of the container shape. However, even for the recent experiments, where $L k$ reaches the value 100 , for low viscousity regime $(\gamma / \omega \geq 0.01)$ the aspect ratio $\Gamma=L / l$ still remains close to unity [31,32]. As the driving increases the pattern becomes time- 
dependent, and in the large containers it looses its spatial coherence via a defect nucleation [33,34. Thus spatio-temporal chaotic behavior rather than the parametric wave turbulence was found in this system far above the threshold.

Comparison of the parametrically generated $S S$ waves in respect to the amplitude saturation mechanism with the Faraday instability. As discussed above the SS amplitude saturation occurs due to the nonlinear attenuation and can be compared with a saturation mechanism of the parametrically driven surface waves investigated recently in details [28, 30]. There are two major differences between these two systems. First, the Faraday ripples have typically a considerably larger reduced dissipation rate $\gamma / \omega$ than the parametrically generated SS waves (at least, two-three orders of magnitude). Second, the marginal condition for the collinear 3W interactions fulfilled with a great precision in the latter system for any wave frequency, due to the linear dispersion relation, while for the Faraday instability the collinear 3W interaction exists only for one specific value of the wave number in the mixed gravity-capillary regime (the Wilton's ripples).

To derive an amplitude equation for the parametrically excited standing surface waves, a classical multiscale method has been applied [28,30]. In the amplitude equation a low order nonlinear term provides a saturation. In a case of spatial isotropy, the waves should be excited in any direction, and the mode selection occurs due to the next nonlinear terms of the form $g_{i j}\left|A_{j}\right|^{2} A_{i}$, where $A_{i}$ and $A_{j}$ are the slowly varying amplitudes of two degenerate unstable modes. For the known coupling coefficients $g_{i j}$, the resulting wave pattern can be found from the amplitude equation [28,30]. The derivation of the amplitude equation for the Faraday waves is greatly simplified in the case of an ideal (inviscid) fluid, where the Hamiltonian approach can be used, and viscous effects then can be treated perturbatively, analogously to the parametrically generated SS waves [22].

For both the parametrically generated surface waves and the SS waves, a major contribution to the wave saturation arises from the 3W interaction [22,28, 29]. The role of triad resonance interactions the amplitude satuartion of low viscous surface waves was first emphasized by Edwards and Fauve 29]. It was explicitly shown by Zhang and Vinals 28 
that for these conditions the dissipation through the excitation of the resonant stable waves at the double frequency is the dominant channel of the amplitude saturation. It was also proved that $3 \mathrm{~W}$ resonant interaction gave the major contribution into the coefficients of the cubic terms of the amplitude equations in this limit.

A particularly relevant case for the comparison with the SS waves is the Wilton's ripples scenario, the parametrically generated surface waves with the frequency corresponding to the collinear $3 \mathrm{~W}$ interaction. The nonlinear term in amplitude equation for Wilton's riples almost vanishes for all angles between interacting waves excluding zero. Zero angle corresponds to self interaction [30]. The characteristic minimal angle of the wave interaction depends on the dissipation rate, $\gamma$. As $\gamma$ decreases the characteristic angle of interactions vanishes, and the number of independent self intracting waves with the same frequency propagating in different directions increases. In the limit of $\gamma \rightarrow 0$, it becomes potentially infinite.

As suggested by Newell and Pomeau [35] an asymptotic planform in this case will be spatially turbulent. It means that spatial correlations of the fields will decay. This state was coined as the 'turbulent crystal'. At a finite dissipation, high symmetry quasiperiodic patterns were observed near the Wilton's ripple condition [32]. The parametrically generated SS waves just above the instability threshold may provide a good experimental realization of the 'turbulent crystal' [35].

The same conclusion about the absence of a pattern formation and a long-range order in the SS waves was made independently by Muratov [22]. Since only the SS waves with the wave vectors in the angle smaller than $\Delta \theta \approx 4 \sqrt{\gamma_{2} / \omega} \ll 1$ interact, the long-range order at larger angles is destroyed, and so the pattern formation is absent 22.

This work deals with the parametrically generated SS waves in cells which aspect ratio, $l / L$, is varied depending on the closeness to $T_{\lambda}$, between 0.1 and 10 ( $L k$ varies between about 200 and 800), whereas the reduced dissipation rate is in the range $10^{-4}<\gamma / \omega<2 \cdot 10^{-3}$. The SS waves have the linear dispersion law which implies $\partial^{2} \omega / \partial k^{2}=0$. It is a marginal case in which the $3 \mathrm{~W}$ interactions are restricted to the collinear waves, whereas waves propagating 
in different directions interact only via the $4 \mathrm{~W}$ processes which are much weaker. All these features make this system particularly interesting to study a highly excited state of the nonlinearly interacting SS waves.

A possibility to observe the fully developed wave turbulence in a system which has two acoustic branches with the linear dispersion law, was discussed theoretically rather extensively for the last several years, and various scalings laws for the spectrum were predicted 17,36 38. If the nonlinear interactions between different acoustical branches are much stronger than the wave self-interactions inside each branch, a shock wave formation is suppressed. Two acoustic noncollinear waves may interact efficiently via waves from another branch. Moreover, it was predicted that the parametric decay processes mainly contribute into generation of an inertial range with algebraic power law spectra. So this system is considered as a promising candidate for an observation and quantitative studies of the fully developed wave turbulence. Thus, the parametric generation of SS waves may be the first step towards creation the developed wave turbulence. The study of this phenomena may give an insight into the nature of the multiwave interaction in this system.

\section{EXPERIMENTAL SETUP.}

According to our estimates, experiments on the parametric generation of the SS waves in the superfluid helium should be performed in the vicinity of the superfluid transition with a temperature stabilization of $\pm 1 \mu K$ or better. Thus, we used a three-stage temperature regulated cryostat with a helium container(experimental cell) as a third stage [39,40]. The detailed description of two experimental cells, where all measurements were performed, follows. There were several specifically designed technical elements that were crucial for success of the experiments. We will describe them below. 


\section{A. Cells.}

To study the parametric instability of the FS waves in the superfluid helium two cells of different geometries were designed. In both of them, we were able to (i) produce the FS wave of a sufficiently large amplitude with known and simple geometry, (ii) measure the FS amplitude, (iii) to measure the SS wave amplitude, and (iv) to test the system by an independent SS wave.

Both cells were short cylindrical resonance cavities for FS. Two round FS capacitive transducers form the endsides of these cavities. Construction and calibration techniques of the FS transducers are discussed in the Sect.IIIB. A diameter of the working part of the transducers was $50 \mathrm{~mm}$. A distance between the transducers was $3.9 \mathrm{~mm}$ and $2.8 \mathrm{~mm}$ in the first and second cells, respectively. Both resonance cavities had rather high quality factors of about $Q=150$. It allowed to obtain sufficiently large FS amplitudes at the resonance frequencies of the cavities and to neglect an influence of all other acoustic modes. The first resonance frequencies for the first and the second cells were weak functions of temperature due to a temperature dependence of the FS velocity in the superfluid helium, particularly in the vicinity of $T_{\lambda}$. In the whole working range of temperatures, the first resonance frequencies lied in the range between 28050 and $28150 \mathrm{~Hz}$ and between 39380 and $39480 \mathrm{~Hz}$ in the first and second cells, respectively. The cell widths were found with a great precision by fitting our experimental data on the temperature dependence of the first resonance frequency in each cell using a single adjustable parameter. The fit was based on the known temperature dependence of the FS velocity 41]. The cell widths were $d=3.833 \mathrm{~mm}$ and $d=2.767 \mathrm{~mm}$ for the first and second cells, respectively.

In the experimental temperature range $2 \times 10^{-4}<\tau<2 \times 10^{-3}$, the SS dissipation length, $l$, varies drastically from $0.2 L$ to $10 L$ for frequencies equal to a half the FS resonant frequency for both cells. That allowed us to study the behavior of the parametrically generated the SS waves in different limits of $l / L$. The main difference in the cell design, however, was in the geometry of bolometers and heaters for SS wave generation and detection. 
The cells were placed into a container filled with about $300 \mathrm{ml}$ of purified $\mathrm{He}^{4}$ and vacuum sealed. This container had a very low heat leak to the rest of the cryostat. A filling capillary was disconnected by a cold valve. That was crucial for a rather high level of temperature stabilization, below $0.1 \mu \mathrm{K}$. To make a thermal contact between cell and a helium bath during the initial cooling, the vacuum isolation space was filled with helium gas at 100 mTorr at room temperature. After the initial cooling, the excange gas was pumped by a sorbtion pump mounted on top flange of a vacuum can. The pump had a very weak thermal leak to the external helium bath [39]. Such a design gave us a possibility to cool the cell in a short time.

\section{B. FS transducers}

In both cells, the FS capacitive transducers similar to that described in Ref. [42], were used. The advantages of using the capacitive transducers are as follow: (i) a relatively high amplitude of an acoustic wave (in comparison to a piezoelectric), (ii)a high sensitivity, (iii) a flat frequency response, and (iv) easy to build. $6 \mu \mathrm{m}$ aluminized mylar film, stretched and glued to an external brass ring, served as one electrode of a capacitor. A sandblasted brass backplate $50 \mathrm{~mm}$ diameter was the second electrode. The capacitance of such a transducer was about $2 \mathrm{nF}$. An amplitude of a driving $a c$ voltage signal was up to $40 \mathrm{Vp}-\mathrm{p}$, and a $d c$ bias up to $300 \mathrm{~V}$. The higher bias increased efficiency and sensitivity of the transducer, but at the same time increased a probability of an electrical breakdown.

Knowing an absolute value of the FS amplitude is very important for studying nonlinear wave dynamics. A method of a transducer calibration using only electrical measurements was first developed by MacLean in 1940 [13]; a general theory of such a calibration can be found in Ref. [44]. In the superfluid helium it was used in Refs. [45,46]. This method is based on a few assumptions: (i) two acoustic transducers are identical and form a resonance cavity; (ii) all acoustic losses happen in a medium and can be measured by the resonance characteristics of the cavity. While the detailed analysis is omitted, the resulting expression 
for the amplitude of the acoustic pressure oscillations at the receiver at the resonance is as follows:

$$
P_{2}=\left(\frac{V_{1} V_{2}}{Z} \frac{2 \rho c_{1}^{2} Q}{S d \omega}\right)^{1 / 2}
$$

where, $V_{1}$ and $V_{2}$ are the voltage oscillations on the first and the second transducers ( the first transducer is the emitter, the second is the receiver), so $V_{1}$ is the $a c$ generator amplitude and $V_{2}$ is the measured amplitude; $Z$ is the electrical impedance of each transducer, $Z=1 / \omega C$, where $C$ is the electrical capacitance; $\rho$ is the density of the medium where acoustic waves propagate ( for helium $\rho \approx 0.145 \mathrm{~g} / \mathrm{cm}^{3}$ at $\lambda$-point); $c_{1}$ is the sound velocity $\left(c_{1} \approx 218 \mathrm{~m} / \mathrm{s}\right.$ ); $Q$ is the quality factor of the resonance cavity, measured from the frequency response of the cavity; $S$ is the surface area of the transducer $\left(S=19.6 \mathrm{~cm}^{2}\right) ; d$ is the distance between transducers ( for the first cell $d \approx 3.9 \mathrm{~mm}$, for the second: $d \approx 2.8 \mathrm{~mm}$ ); and $\omega=2 \pi f$ is the frequency of the sound wave. All values, $V_{1}, V_{2}$ and $\omega$, correspond to the resonance.

The measurements of an acoustic sensitivity of the transducers, $M$, and an efficiency of the acoustic resonator, $E$, were made for both cells:

$$
\begin{aligned}
M & =\frac{\text { Measured ac signal }\left[\mathrm{V}_{\mathrm{rms}}\right]}{\text { Pr essure oscillations }\left[\mathrm{Pa}_{\mathrm{rms}}\right]}, \\
E & =\frac{\text { Pr essure in the resonance }\left[\mathrm{Pa}_{\mathrm{rms}}\right]}{a c \text { voltage amplitude on the generator }\left[\mathrm{V}_{\mathrm{p}-\mathrm{p}}\right]} .
\end{aligned}
$$

It was found that $M$ and $E$ did not depend on temperature in the experimental range and with the given accuracy. Temperature changes of the FS resonance frequency, of the quality factor of the acoustic cavity (FS attenuation), of the amplitude of the signal at the resonance and of the helium density can be neglected.

The measured sensitivity of the transducers in the first cell was $M_{I}=1.56 \cdot 10^{-5} \mathrm{~V}_{r m s} / \mathrm{Pa}$, and in the second cell $M_{I I}=1.75 \cdot 10^{-5} \mathrm{~V}_{r m s} / \mathrm{Pa}$. The efficiency of the resonance cavities were: $E_{I}=3.5 \mathrm{~Pa} / \mathrm{V}_{p-p}$, and $E_{I I}=7.5 \mathrm{~Pa} / \mathrm{V}_{p-p}$. 


\section{The First Cell.}

The first experimental cell (Fig.3) was designed as partially reflecting for the SS waves in a horizontal plane. Two SS bolometers and two heaters, evaporated on $22 \mathrm{~mm}$ diameter glass substrates, were placed diametrically opposite to each other (one bolometer is opposite to one heater), and the two pairs were mounted perpendicular to each other. Each heaterbolometer pair separated by $54 \mathrm{~mm}$ apart, formed a resonant, partially open cavity for the SS waves with resonances from 12 to $40 \mathrm{~Hz}$ apart depending on temperature. Possible incidental effects of these resonances on the experimental observations will be discussed below. Four glass substrates covered together about half of the cell perimeter; the remaining part was open. Several layers of crumpled paper were put around the cell to absorb the FS and SS waves. To decrease the acoustic crosstalk between the FS transducers, each glass substrate was attached to the side of the cavity through a layer of thick filter paper. The parametrically generated SS waves were partially reflected from the bolometer and the heater substrates, and partially escaped from the cell being absorbed by the paper around the cell.

\section{Flat bolometer and heater.}

Bolometers were $40 \mu \mathrm{m}$ wide superconducting $\mathrm{Au}-\mathrm{Pb}$ stripe in the form of a round serpentine pattern of diameter $d_{b}=2 \mathrm{~mm}$, evaporated on a cover glass (Fig.⿴囗十). The technology of such bolometers was early developed in our laboratory [四,40]. The figure of merit of the bolometers was about $\alpha=R^{-1}(\partial R / \partial T) \approx 100 \mathrm{~K}^{-1}$ in a zero magnetic field.

Since $\lambda / d_{b} \ll 1$ in the working temperature range (the SS wave length $\lambda$ changed from 0.1 till $0.5 \mathrm{~mm}$ ), these bolometers were sensitive mainly to waves incident almost perpendicular to the them within the angle of $\sim \lambda / d_{b}$ to the normal direction, i.e., from 0.05 to 0.15 radian. Thus they were able to detect the SS waves generated only in the central part of the cell.

A heater was produced by evaporation of a thin gold film on a $22 \mathrm{~mm}$ diameter glass substrate. Contacts were made of a thick gold film( $\sim 150 \mathrm{~nm})$. A heater resistance was 18 
$\Omega$ at liquid helium temperatures.

\section{The Second Cell.}

As the experiment showed, a partial reflection of the SS waves from the bolometers and the heaters caused certain difficulties in an interpretation of the experimental results and their comparison with the theory particularly above the instability threshold. Thus, to resolve these problems, the second cell with the non-reflecting bolometers and the heater was designed. The development of a new type of the bolometers and the heater was the main technological challenge in the cell design [47].

\section{Fiber bolometer.}

The bolometers were prepared on glass fibers which diameter was much smaller than the SS wave length $0.1<\lambda<0.5 \mathrm{~mm}$. A superconducting gold-lead (2:1 composition) film of an approximately $20 \mathrm{~nm}$ thickness was evaporated on $8 \mu \mathrm{m}$ diameter glass fiber. While the detailed description the bolometers can be find in Ref. [47, here we summarize their main features:

(i) No reflections. The bolometers with no reflection first were proposed in Ref. 48, where they were made by coating glass fibers of diameter $d \approx 10 \mu \mathrm{m}$ with a layer of gold and tin. However, such bolometers did not have a high enough figure of merit and were not designed for measuring near the superfluid transition temperature. The diameter of the glass fibers in our case was $8 \mu \mathrm{m}$, close to that reported in [48], and much less than a SS wave length. A SS wave reflection from such bolometers was negligible.

(ii) Uniform angular sensitivity. Since the diameter of the fiber bolometer was much smaller than the SS wave length, the bolometer was sensitive to all SS waves propagating in the plane perpendicular to its axis, in contrast to a flat bolometer.

(iii) High figure of merit. The figure of merit of these bolometers was $\alpha=R^{-1}(d R / d T) \approx 30$ $\mathrm{K}^{-1}$, that was comparable with the best flat bolometers 40. 
(iv) Working temperature range is near $\lambda$-point. The bolometers were built on a basis of the same type of superconducting gold-lead alloy that was used in works [4,40] and [19].

(v) Magnetic field and current tunability. A capability to tune $T_{c}$ of the bolometers by varying the bias current, while still maintaining a high figure of merit, was essential for the use of these bolometers in our experiments. The experimental cell contained eight bolometers operating simultaneously with a high sensitivity. Since all the bolometers in the experimental cell saw the same field from an external solenoidal magnet, it was the individual bias current which was adjusted to bring each bolometer's $T_{c}$ to a helium working temperature.

(vi) Long time room stability. The superconducting layer was covered by $40 \mathrm{~nm}$ of $\mathrm{MgF}_{2}$ that decreases a rate of an oxidation at a room temperature. Since a technology of preparation of the bolometers and their mounting in the cell required a high accuracy and took a long time at a room temperature, it was necessary to prevent a fast oxidation of the superconductor. The bolometers could survive room temperature conditions during many hours and almost did not change their properties after a month being in a vacuum desiccator.

In order to have a possibility to measure an angular distribution of the SS waves in the cell plane, eight bolometers were mounted around the cell at equally spaced intervals on the circumference of the cell (Fig.5). The number of the bolometers was defined by a maximal number of coaxial cables permitted by a given wiring setup. Unfortunately, only five among eight bolometers worked after cooling.

The bolometers were placed in semi-cylindrical (1.5 mm radius) groves machined in two rings made of G-10, mounted around the FS transducers. Such design allowed to electrically isolate the fiber bolometers from the FS transducer housing made of brass, and to put them as close as possible to the working area of the transducer membrane, so that a SS wave path without a FS pumping was minimal. To decrease the electrical pick-up and crosstalk by the bolometers, all the wiring was made by coaxial cables down to the cell and twisted pairs inside the cell. For the same purpose, a thin copper wire $(0.05 \mathrm{~mm})$ was soldered parallel to the bolometer fiber and connected to the bolometer contact on one side of the cell. A 
twisted pair was connected to other bolometer contact and this wire. In this way an area of electrical loop which could act as an antenna for a crosstalk, was decreased.

\section{Fiber heater.}

The nonreflecting SS emitter-heater was constructed from 31 glass fibers of $8 \mu \mathrm{m}$ diameter spaced $1 \mathrm{~mm}$ apart. A fiber length was equal to the cell width, $3 \mathrm{~mm}$. The fiber grid was covered by $\approx 80 \mathrm{~nm}$ of $\mathrm{Cr}$. This grid produced a plane wave at a distance larger than a fiber spacing, $1 \mathrm{~mm}$, and less than a grid width, $30 \mathrm{~mm}$. The incident SS waves coming from the cell, were not reflected from the heater.

The details of a fiber bolometer and heater fabrication, mounting, testing and all characteristics can be found in Ref. [15,47].

\section{E. SS phase locking thermal stabilization.}

To improve a thermal stabilization of the experimental cell, a dedicated SS phase locking (SSPL) thermometer was built. The idea of such thermometer was proposed and experimentally verified by H. Davidowitz [40,49]. The SS velocity is a sharp function of the closeness to $T_{\lambda}$. So, small temperature fluctuations in the cell lead to strong fluctuations of the phase of the SS sine wave continuously emitted from the heater and measured by the bolometer. The phase signal is sent to a temperature stabilization loop.

Together with the experimental cell, a separate cell for SSPL thermal stabilization was placed inside the helium container. A radio frequency wave guide (cross-section $3.5 \times 7 \mathrm{~mm}^{2}$, length $60.6 \mathrm{~mm}$ ) was used as a resonance cavity. One side of the cavity was formed by a flat heater, evaporated on a piece of glass substrate. The fiber bolometer, attached to a flat G-10 plate, was mounted on the opposite side.

The phase of the detected SS signal relatively to the phase of the emitted signal was measured by a lock-in amplifier. Such a thermometer did not allow to measure the absolute value of the temperature in the cell but provided a very high temperature stability. The 
temperature can be measured by measuring a time propagation of the SS pulse in the same cell.

The sensitivity of the thermometer increases while approaching $T_{\lambda}$. The best result on the temperature stabilization of $0.1 \mu \mathrm{K}$ rms was obtained at the reduced temperature $\tau=5 \times 10^{-5}$ with a SSPL frequency $f_{s}=970 \mathrm{~Hz}$.

\section{F. Signal acquisition.}

We developed a new technique to measure the SS spectra in a narrow band around the central $\Omega / 2$ peak. We also describe a home-made eight-channels preamplifier with a lock-in amplifier for a simultaneous signal acquisition from eight bolometers.

\section{Spectrum measurements.}

As follows from the theory and found by our experiments, the SS spectra should be very narrow and centered at half of the FS frequency, $F / 2=2 \pi \Omega / 2$. For temperatures close to $T_{\lambda}$, the width of a single peak around $F / 2, \Delta$, should be much less than the SS attenuation, $\Delta / \gamma_{2} \ll 1$, where $\gamma_{2} \sim 100 \mathrm{~Hz}$. And for temperatures far from $T_{\lambda}$, the SS spectra should consist of two peaks with a distance apart of $\delta f=\left(c_{2} / c_{1}\right)^{2} F / 2$, that is of the order of a few Hertz.

Thus, an idea of the measurement technique is to measure a signal from the bolometer by a lock-in amplifier with a reference frequency exactly equal to a half the FS frequency, $F_{p} / 2$, to record time dependent signals from a lock-in output and then by, calculating their Fourier spectrum, to rebuild the real spectra in a narrow bandwidth around $F_{p} / 2$. The measuring spectral width should be smaller than an inverse lock-in integration time constant. Then as shown in Ref. [15], the relation between the Fourier component of a real, timedependent signal at the lock-in amplifier input, $A\left(\omega_{0}+\delta\right)$, and the Fourier component of

a complex, slowly changing signal at the lock-in amplifier output, $Z(\delta)$, is given by the following expression at the gain equal one [15]: 


$$
Z(\delta)=\frac{A\left(\omega_{0}+\delta\right)}{\sqrt{1+(\delta t)^{2}}} \exp ^{i \arctan (\delta t)},
$$

where $\delta=\omega-\omega_{0}$ and $t$ is the lock-in amplifier integration time. Thus, both the phase shift and the amplitudes of spectral components of the complex output signal should be corrected according to Eq.(27).

Both components of the lock-in complex output signal were sampled and digitized at a rate $F_{\text {samp }}$ during an interval $T_{\text {meas }}$. As a rule we used $F_{\text {samp }}=16 \mathrm{~Hz}$ and $T_{\text {meas }}=128$ sec. Then the complex Fourier transform of both components of the signal provided the SS spectrum in a narrow window, $\pm 8 \mathrm{~Hz}$, around $F / 2$ with resolution of $1 / 128 \mathrm{~Hz}$.

\section{Multichannel lock-in and preamplifier.}

For the second experiment with 8 bolometers, a dedicated low noise preamplifier and a lock-in amplifier were built. The low noise, wide bandwidth preamplifier with constant gains $\left(\mathrm{x} 10^{3}\right)$ had nine channels: eight for the cell bolometers and the ninth for the SSPL bolometer. A noise level in a frequency range from $100 \mathrm{~Hz}$ to $100 \mathrm{kHz}$ did not exceed $2 \mathrm{nV} \mathrm{Hz}-1 / 2$. It was found that a main noise source was ratherthe bolometers than the preamplifier. The preamplifier had also an adjustable simple $d c$ current source $(50-150 \mu \mathrm{A})$, for a bolometer biasing. The preamplifier was located very close to the cryostat terminal box and had an independent battery power source $( \pm 12 \mathrm{~V})$.

Signals from 8 preamplifiers were fed to a home-made 8-channel lock-in amplifier. The lock-in amplifier had a reference at a double frequency compared to the frequency of the measuring signal. This unique property was very convenient for detection of the SS signal at a half the FS frequency. The input signal was multiplied by two square waves at a half the FS frequency with phases shifted on $\pi / 2$ and integrated during a given time. The two shifted square waves were easy to generate out of the reference square wave signal taken from the $S Y N C$ output of the FS generator, by logical TTL manipulation. Low frequency signals from sixteen lock-in outputs were send to a A/D computer card. 


\section{Instrumental setup.}

A general scheme of our measurements is shown in Fig.6. The FS transducer was pumped by an ac signal from a function generator $(\mathbf{G 1})$ [50]. The generator can give a sine wave with amplitude up to $40 \mathrm{~V}_{p-p}$. The FS amplitude was measured by an Ithaco lock-in amplifier [51] referenced from the "SYNC" output of the generator. The amplitude reading was digitized and sent to the computer via a Keithley 197 DMM (V). The SS measuring circuits required a biasing current of about $100 \mu \mathrm{A}$. The dedicated 8-channel preamplifier with the biasing current was designed and built. Each bolometer was connected to the preamplifier box by a single coaxial cable which transferred both the bias current and the measuring signal. All coaxial cables were grounded at a single point on the cell level.

The output signals from the preamplifiers were sent to the 8-channel lock-in amplifier, that takes its reference signal from the FS generator. The lock-in amplifier was designed to have the reference frequency twice larger than the frequency of the signal of interest. So to measure the parametric instability signal near a half the FS frequency, the signal from "SYNC" output of the FS signal generator was directly fed to the lock-in "Reference" input. In the first experiment a standard lock-in amplifier was used. Such a lock-in required a reference signal equal to a half the FS frequency. A small home-made frequency divider was introduced between the generator "SYNC" output and the lock-in "Reference" channel.

For the experiments on a response of the interacting wave system to an independent SS wave, and for a bolometer check, independent SS emitters-heaters were mounted in the cell. The heater was connected to a Synthesized Function generator DS345 [52] (G2) that can produce both a burst signal for measuring pulse arrival time and a continuous sine wave. Quartz clocks of both generators G1 and G2 were synchronized. This option was essential for the experiments on simultaneous pumping of the FS and SS waves when a SS wave with a frequency shifted from a half the FS frequency needs to be emitted. 


\section{EXPERIMENTAL RESULTS AND COMPARISON WITH THEORY.}

Presentation and discussion of the experimental results are divided into three major sections: (i) linear properties of the parametric instability; here we discuss all threshold related phenomena: the temperature dependence of the threshold and finite size effects, and the splitting of the SS spectrum just above the threshold; (ii) nonlinear behavior of the parametrically excites SS waves above the threshold: the SS amplitude saturation mechanism, the broadening of the SS spectrum above the threshold, and the statistics of the SSwave amplitudes; and (iii) the experiments on a simultaneous FS and SS pumping that include an observation of an acoustic phase conjugation and a giant SS amplification above the threshold.

To avoid further misunderstanding we need to comment words "linear" and "nonlinear". All our results are about the wave interactions in the superfluid helium that are in general nonlinear phenomena, however, when we use the word "linear" we mean linear in the respect to the SS wave amplitude. Correspondingly we use the word "nonlinear." Nonlinear phenomena, by our classifications, are those where the interactions between SS waves play a crucial role.

\section{A. Parametric instability: linear properties.}

\section{Experimental results}

Measurement of the threshold of the parametric instability.

The experiments to define the parametric instability threshold of the FS waves in the both cells were performed in the same way. At a fixed temperature, FS was generated at a frequency $\Omega$, which was chosen to be the first resonance frequency of the cavity. For a given FS amplitude, a SS amplitude was measured by the bolometers. Typical plots of the time averaged SS intensity in the vicinity of $\omega=\Omega / 2$ as a function of the driving FS amplitude for the first and second cells are shown in Fig.7. Averaging time intervals for the first and 
second cells were 128 and 32 seconds, respectively. A well defined threshold for the driving amplitude, at which the SS intensity first exceeds twice a background noise level, exists for each curve in Fig.7.

One can notice a striking difference between the SS intensity dependences for the first and second cells. In the first cell above the instability threshold, the SS intensity plot exhibits rather large fluctuations as a function of the FS amplitude. It is important to point out here, that the SS amplitude in this cell also strongly fluctuates in time. The characteristic time of these fluctuations was particularly long near the onset where the time intervals between isolated spikes could be as long as 1000 seconds (which was the maximal sampling interval). This time is much larger than all characteristic times in the problem. These fluctuations limit the resolution of the threshold determination and hinder quantitative studies of the amplitude dynamics above the onset. Nevertheless, they do not change the main features of the phenomena discussed below. The SS intensity plots for the second cell are more regular.

The temperature dependences of the FS threshold amplitude for both cells are presented in Fig.8. The solid lines are theoretical curves which are plotted without any fitting parameter. As we already discussed in the Section [1], there are two important factors which should be taken into account. First, one should consider the generation of the SS waves by the standing FS waves in a resonance cavity rather than by the propagating ones. Second, the cell size should be compared with the SS attenuation length that drastically depends on temperature.

SS spectra just above the threshold.

Another result found at the instability threshold, was two types of the SS power spectrum that were observed in different temperature regions. Examples of two typical SS power spectra at the reduced temperatures $\tau=2.19 \times 10^{-4}$ and $\tau=6.52 \times 10^{-4}$ in the first cell , are shown in the insets of Fig.9. The first spectrum has a single sharp peak exactly at frequency $\Omega / 2$, while the second one, obtained further away from $T_{\lambda}$, exhibits two peaks equally separated from $\Omega / 2$. The left peak at the lower frequency, $\Omega / 2-\delta f$, is always larger than the right one at the frequency $\Omega / 2+\delta f$. 
The temperature dependence of the frequency peak shift is shown in Fig.9. Closer to $T_{\lambda}$, the spectrum has just one peak at the frequency $\Omega / 2$ that corresponds to the symmetric decay of FS. Further away from $T_{\lambda}\left(\tau>6 \times 10^{-4}\right)$, the spectrum shows two peaks equally separated from $\Omega / 2$. A crossover from one type of the spectrum to another occurs in the reduced temperature interval $4 \times 10^{-4}<\tau<6 \times 10^{-4}$, where both the central peak and the pair of the separated peaks coexist in the spectrum.

The two peak spectra are always asymmetric: the left peak corresponding to the lower frequency is always higher that the right one.

Features of the second cell spectra

The geometry of the second cell differs from that of the first cell, and this causes some differences in a SS response. The most striking fact was that the SS spectra in the second cell were different on different bolometers and had also different frequency shifts from an exact $\Omega / 2$ value. A typical SS spectrum just above the onset of the parametric instability consists of a single instrumentally sharp peak shifted from $\Omega / 2$. The peak shifts are different on different bolometers and depend on temperature. Their temperature dependences are shown in Fig.10. The bolometer positions in the cell are shown in the inset of Fig.10. The bolometers 2 and 8, that are above the dotted line, connecting the bolometers 3 and 7 , have negative frequency shifts. The bolometers 4 and 5, located below the line, have positive frequency shifts ( the frequency shift for the bolometer 5 was positive but the SS signal was very noisy). The bolometer 7 has almost no frequency shift.

\section{Comparison between the experiment and theory.}

In order to explain the experimental results on the temperature dependence of the instability threshold and the spectrum splitting as well, let's first estimate the range of variations of important parameters in the problem, namely, the sound velocity ratio, $\eta=c_{2} / c_{1}$, the ratio of the SS dissipation length to the horizontal size of the cell, $l / L$, and the ratio of the frequency splitting to the SS dissipation rate, $\Omega \eta^{2} / \gamma_{2}$. 
In the working temperature range, $10^{-4}<\tau<2 \times 10^{-3}, \eta$ varies between 0.006 and 0.019. In the same temperature range, $l / L$ changes from 11 till 0.2 in the first cell, and from 5.5 till 0.1 in the second one. The ratio $\Omega \eta^{2} / \gamma_{2}$ varies between 9 and 0.05 in the first cell, and between 6 and 0.04 in the second one.

Temperature dependence of the FS threshold amplitude.

The experimental data on the temperature dependence of the FS threshold amplitudes of the parametric instability for both cells are presented in Fig.8 together with various theoretical curves. The general expression for $a_{t h}$ from Eq.(11) is used. Curves $\mathbf{A}$ in both plots show the lowest possible FS threshold amplitude which corresponds to an infinite cell with the resonance cavity factor $\zeta=1 / 2$. Two conditions should be satisfied in order to apply this criterion: (i) $l / L \ll 1$, and (ii) $\Omega \eta^{2} / \gamma_{2} \ll 1$. It occurs in the close vicinity to $T_{\lambda}$. At $\tau=10^{-4}$ the ratios in the first and second cells are the following:

$$
\begin{aligned}
& \text { I. } \quad l / L=0.21, \quad \Omega \eta^{2} / \gamma_{2}=0.052 \\
& \text { II. } \quad l / L=0.10, \quad \Omega \eta^{2} / \gamma_{2}=0.036
\end{aligned}
$$

The maximal values of the FS threshold amplitude are realized in a finite cell without SS reflections from the boundaries, $r=0$, and with the spectrum splitting, i.e., at $\zeta=1 / \sqrt{2}$ - curves $\mathbf{B}$ in the both figures. This opposite limit is reached at the conditions: (i) $l / L \gg 1$, and (ii) $\Omega \eta^{2} / \gamma_{2} \gg 1$, which are satisfied far from the superfluid transition. For example, at $\tau=10^{-3}$ one finds

$$
\begin{gathered}
\text { I. } \quad l / L=4.8, \quad \Omega \eta^{2} / \gamma_{2}=2.8 \\
\text { II. } \quad l / L=2.3, \quad \Omega \eta^{2} / \gamma_{2}=2.0
\end{gathered}
$$

The transition from one regime to another occurs within the following experimental temperature range in the both cells:

$$
\begin{gathered}
l / L=1: \quad \text { I. } \tau \approx 3.1 \times 10^{-4}, \quad \text { II. } \tau \approx 5.3 \times 10^{-4}, \\
\Omega \eta^{2} / \gamma_{2}=1: \quad \text { I. } \tau \approx 5.5 \times 10^{-4}, \quad \text { II. } \tau \approx 6.8 \times 10^{-4} .
\end{gathered}
$$


The experimental data for the FS threshold amplitude as a function of the reduced temperature in the first cell lie between two theoretical limits (Fig.8.I): curve A represents the threshold in an infinite cell without the spectrum splitting $(r=1, \zeta=1 / 2)$, and curve $\mathbf{B}$ shows the threshold in a finite cell without reflection $(r=0)$ and with the spectrum splitting $(\zeta=1 / \sqrt{2})$. An additional curve $\mathbf{C}$ is plotted with the reflection coefficient $r=0.7$ and the spectrum splitting $(\zeta=1 / \sqrt{2})$. It fits the data rather well for the most part of the experimental temperature range.

For the temperature range close to $T_{\lambda}$, the experimental points lie above the curve A, that presents the threshold in an infinite cell. A reasonable explanation can be the following. In the limit of $\Omega \eta^{2} / \gamma_{2} \ll 1$, the excited SS waves have no spectra splitting and propagate in the cell plane. The conditions of the SS generation by the FS standing waves vary along the vertical axis. Indeed, in the standing FS waves the regions of maximal amplitudes of pressure and velocity are separated in space. It was shown in [22], that it is the pressure oscillations in the FS waves that are responsible for the SS generation. So, the most preferable conditions for the SS wave generation are in the antinodes of the pressure in the FS standing wave. Thus, the SS waves are excited in narrow layers near the FS transducer surfaces and propagate along them. At the same time, the SS bolometers are located in the first cell near the central cell plane and are not sensitive to the SS waves propagating near the transducer surfaces. It means that the bolometers can only detect the SS waves well above the onset, and the real threshold value can not be measured.

The experimental data for the second cell agree with the theoretical predictions rather well (Fig.8.II). All the experimental points lay between two curves B and D. The curve D presents the threshold for the cell with the zero reflection coefficient, $r=0$, and the numerical factor $\zeta=1 / 2$, that corresponds to no spectra splitting. For small $\tau$, the experimental points are fitted by the curve $\mathbf{D}$, and for large $\tau$-by the curve $\mathbf{B}$. The crossover happens at the intermediate temperatures.

Splitting of SS waves spectra.

The experiments in the first cell exhibit different types of the SS spectrum in the different 
temperature ranges that is well explained by the considerations of the finite size geometry in the direction of the FS propagation and by the discreteness of the $z$-component of the SS wave vector (see Sect.IIB3). In the experimental temperature range, the attenuation of the SS waves changes sharply, so the both limits $\Omega \eta^{2} / \gamma_{2} \ll$ and $\gg 1$ can be observed. Then indeed, if the $\Omega \eta^{2} / \gamma_{2} \ll 1$, that occurs closer to $T_{\lambda}$, the discreteness of the resonance states is smeared out. This case corresponds to the symmetric decay with a single peak in the SS spectra. The opposite case $\Omega \eta^{2} / \gamma_{2} \gg 1$ leads to the discrete resonance states and two peaks in the SS spectrum. The temperature dependence of the frequency shift of the peaks $\delta f= \pm(F / 2) \eta^{2}$ is plotted as dashed lines in Fig.9 (here $F=\Omega / 2 \pi$ ). It describes the experimental data rather well. Two peaks spectrum corresponds to the decay process in which one of the SS waves propagates normally to the FS wave direction, and both components of the FS standing waves contribute to the parametric excitation. On the same plot, the temperature dependence of the SS attenuation rate, $\gamma_{2} / 4 \pi$, is also shown (Fig.9, dotted line). For the temperature range, where two peaks are observed, the following inequality is satisfied: $\gamma_{2} / 4 \pi<\delta f$, while in the region of a single peak, the relation is opposite: $\gamma_{2} / 4 \pi>\delta f$.

The large difference in the measured peak amplitudes is probably due to different SS modes corresponding to each peak. The peak with a lower frequency corresponds to the zero eigenmode along vertical axis, that has a symmetric distribution with respect to the central plane. The peak with a higher frequency corresponds to the first mode, that is antisymmetric. The measured signal on the bolometer is a result of an interference. The bolometer is symmetric with respect to the central plane. So it can detect the antisymmetric mode only in the case when the bolometer location deviates from the central plane symmetry. Therefore, the higher frequency signal should have much lower amplitude.

Features of SS spectra in the second cell.

The spectra of the SS waves in the second cell differ greatly from the SS spectra in the first cell. They are much more irregular. These irregularities in the spectra do not change the temperature dependence of the FS threshold amplitude but do smear out the SS spectral 
splitting effect, observed in the first cell.

There are two main factors that make two cells so different. First, there is no reflection of the SS waves in the second cell in contrast to the first one. Second, the bolometers in the cells have very different angular sensitivity as described in the Sect.ПII and IIID.

The striking result on the frequency shift of the SS spectrum peaks just above the threshold of instability (Fig.10) can be explained by anisotropy of the FS acoustic field which had a non-zero component in the horizontal plane. The FS traveling waves could propagate in the direction shown by the arrow in the inset (Fig.10). This could result from asymmetric FS reflection from the cell boundaries. Both of the wave vectors of the FS standing wave components could be slightly tilted. In such a case, the SS spectrum measured on a bolometer, should be shifted from exact $F / 2$ value by

$$
\Delta f=F\left(\frac{c_{2}}{c_{1}}\right) \cos \alpha
$$

where $\alpha$ is the angle between the FS wave vector in the horizontal plane and the direction of the SS wave propagation which is along the radial direction in the cell plane. So, for the bolometers 2 and 8 one has $\cos \alpha<0$, for the bolometers 4 and 5 one has $\cos \alpha>0$, and for the bolometer 7 one finds $\cos \alpha \approx 0$.

As follows from Eq.(28) the frequency shift due externally broken isotropy in the cell plane is proportional to $c_{2} / c_{1}$. This dependence is different from the dependence due to spectral splitting considered above, where $\delta f \sim\left(c_{2} / c_{1}\right)^{2}$. Since the frequency shift due to anisotropy is larger than the spectral splitting, that is probably the reason why the latter effect was not observed in the second cell.

The experimental data on the frequency shift in the second cell were fitted by Eq.(28). The dashed lines in Fig.10 show the result of the fitting for each pair of the bolometers with a single adjustable parameter $\alpha$. The values of angles obtained from the fit for different bolometers, are the following: $\alpha_{2} \approx \alpha_{8} \approx(90+1.6) \mathrm{deg}, \alpha_{4} \approx(90-1.4) \mathrm{deg}, \alpha_{7} \approx 90 \mathrm{deg}$.

However, in spite of these nuisant effects it is still possible to characterize nonlinear behavior of the SS waves above the threshold in the second cell. 


\section{B. Nonlinear properties of SS waves above the threshold.}

\section{Nonlinear saturation mechanism.}

As the FS amplitude increases above the threshold of the parametric instability, the SS amplitude starts to grow exponentially in time. One of the central questions in this respect is a nature of a mechanism of a SS amplitude saturation. As we discussed in Sec. [IC1, the theory [22] suggests that the $3 \mathrm{~W}$ resonance interaction is responsible for the saturation. According to that theory, the SS wave intensity above the threshold is defined by the nonlinear attenuation and has the following dependence on the control parameter of the instability: $I_{2} \sim A / A_{t h}-1$, see Eq.(17). Here $A$ and $A_{t h}$ are the FS amplitude and its threshold value, respectively. This scaling is very different from that predicted and observed for spin waves, where the amplitude saturation occurs due to the dephasing mechanism. Then the spin wave intensity depends on the control parameter as $I \sim \sqrt{\left(A / A_{t h}\right)^{2}-1}[10$.

The experiments in the first cell did not provide a clear cut answer about the saturation mechanism. An average intensity, particularly at large values of the control parameter, $\epsilon=A / A_{t h}-1$, fluctuated so strongly with the control parameter, that it was impossible to establish the intensity dependence on $\epsilon$ (see Fig.7.I). A possible mechanism for such strong fluctuations will be discussed below.

The data on the SS intensity as a function of $\epsilon$ in the second cell can be analyzed quantitatively. The typical plot of the SS intensity in a wide range of the FS amplitude variation is shown in Fig.11. This plot is undoubtfully exhibits linear dependence on the control parameter $I_{2}=g_{\text {exp }} \epsilon$, where $g_{\text {exp }}$ is the parameter of the linear fit. It was possible to measure this parameter in a wide temperature range in the vicinity of $T_{\lambda}$ only for two bolometers, 4 and 8 . The dependences of $g_{\text {exp }}$ on the reduced temperature for these bolometers are presented in Fig.12. For the reduced temperature $\tau=3.3 \times 10^{-4}$ one finds $g_{\text {exp }} \approx 10^{-6} \mu W / \mathrm{cm}^{2}=10^{-8}\left(\mathrm{~W} / \mathrm{m}^{2}\right)$.

Thus, the data verified the theoretically found functional dependence of the SS wave 
intensity above the threshold on the control parameter presented by Eq.(17). However, we found significant quantitative discrepancy both in the value of the coefficient in this expression and its temperature dependence (see Sec.IC1).

In order to compare the experimental value of $g_{\text {exp }}$, we would like, first, to make comments about a dimensionality of SS wave packets in the first and the second cells. In both cells, the SS waves propagate in the plane of the FS transducers. That defines 2D geometry of wave propagation. In the first cell dimensionality of the wave pattern may be reduced to 1D, due to boundary conditions. The resonance reflections from the bolometer and heater substrates may select 1D pattern of the wave propagation. This effect should be observed further away from $T_{\lambda}$. Closer to $T_{\lambda}$, the dissipation length becomes very short, and the role of the boundaries diminishes. In the second cell with the non-reflecting boundaries the dimensionality of the wave packets should be 2D. A nonlinear pattern selection may change it to $1 \mathrm{D}$, however, we do not see any evidence of such a phenomenon.

Even for the lowest theoretically predicted value of $g$ that corresponds to $1 \mathrm{D}$ case, we found a large discrepancy with the experiment. For the temperature $\tau=3.3 \times 10^{-4}$, the experimental value $g_{\text {exp }}$ is 450 times smaller than theoretically predicted one. In $2 \mathrm{D}$ case the discrepancy even higher by the factor $\sqrt{\omega_{0} / \gamma_{0}}$. Comparison of the results in two cells does not improve the situation. While the dimensionality of the first cell should be lower, and $g$ should be smaller correspondingly, one can see from the plots in Fig. 7 .I that the average slope of the SS intensity vs $\epsilon$ in the first cell, particularly at small values of $\tau$, is an order of magnitude larger than in the second cell. The inconsistency exists not only in the data for the different type of the bolometers in two cells but even for two different bolometers of the same type in the same cell (see data on two bolometers in Fig.12). Moreover, as it is clear from Fig.12, a different functional dependence of $g_{\text {exp }}$ on the reduced temperature was observed for two bolometers: for the bolometer 4 one finds $g_{4} \sim \tau^{3}$, and for the bolometer 8 one has $g_{8} \sim \tau^{4}$. Both these power laws are very different from the theoretically predicted $g \sim \tau^{0.33}$ 22.

The reason for such great quantitative discrepancies between the theory and the experi- 
ment, and also inconsistencies of the results on the different bolometers and in two cells may be explained by the following arguments. There are two unknown factors that can influence the absolute value of the SS amplitude measurements: (i) an inhomogeneity of the bolometer sensitivity along the fiber, and (ii) a nonuniformity of a profile of a SS wave packet across the cell height. The consequence of the latter factor on the measurements of the instability threshold in the first cell has been already discussed above (Sect IV A 2). A nonuniform spatial structure of a SS wave packet, that was not taken into account by the theory [22], maybe another possible explanation of the quantitative discrepancy. For the sufficiently small $\tau$ the SS wave spectrum exhibits only one central peak (see Sect.IVA2). Then the SS waves propagate almost in the cell plane. However, the SS wave packet has nonuniform amplitude distribution in the vertical direction. As was discussed above, the most preferable conditions for the SS wave generation are in narrow layers near the FS transducers, because the amplitude of the pressure oscillations there has a maximum. Moreover, two SS waves, propagating along two transducer surfaces and along different directions in the transducer plane maybe almost incoherent and produce a random destructive interference on the bolometers. All these factors can drastically reduce the measured SS intensity.

The measurements of the SS intensity require a bolometer calibration. In our cell, it was possible to make only a static bolometer calibration, namely to measure the bolometer resistance vs the cell temperature. For experiments with the SS plane wave such calibration was sufficient to get correct numbers [3]. However, in the experiment on the SS parametric generation the distribution of the SS amplitude between the FS transducers is unknown. If the SS wave packet profile across the cell is far from planar wave and the SS waves from different directions are incoherent, it is easy to get the measured signal ten and even more times smaller than a real amplitude of the SS wave for bolometers with inhomogeneous sensitivity distribution along the fiber. This gives correspondingly more than two orders of magnitude error in the SS intensity measurements. Then bolometers with different inhomogeneity can measure different SS amplitudes for the wave packet. The discrepancy between different bolometers can depend on temperature because both the bolometer sensitivity distribution 
and the SS wave amplitude profile can be a function of temperature.

Thus, in spite of the fact that the measurements of the SS intensity above the instability threshold in the second cell do not provide a possibility of quantitative verification of the theoretical predictions on the coefficient $g$ from Eq.(17), these experiments do provide information about the power dependence of the SS intensity as a function of the control parameter. In this way, the experimental results do verify the theoretical prediction that the nonlinear attenuation is the main mechanism of the SS amplitude saturation. We can not exclude the possibility that the major mechanism of SS amplitude satuartion is different from the nonlinear attenuation, and the linear dependence of the SS amplitude is the result of a coincidence of many other factors discussed above. However, this is much less probable and we have no arguments to prove it.

\section{Broadening of the SS spectrum above the threshold.}

It was already pointed out, that the dynamic nonlinear behavior of the parametrically generated SS above the threshold is highly intermittent. Such intermittent behavior differs greatly from a known example of the parametrically excited surface waves [11 (see Sect.IID). In particular, the difference with pattern forming systems manifests itself in low frequency fluctuations of the SS amplitude (Fig.13). The characteristic time of the fluctuations is much longer than the relaxation time of SS waves, $\gamma_{2}^{-1}$, and their traveling time, $L / c_{2}$. The behavior similar to this was observed in some cases of the parametrically excited spin waves [10.

In the whole experimental temperature range, the SS frequency spectra just above the threshold consist of one or two instrumentally narrow peaks that broaden continuously as the control parameter increases. In spite of the fact that the SS frequency spectra exhibit qualitatively similar behavior in the entire temperature range, it is possible to analyze them quantitatively only in a narrow range of the reduced temperatures between $2 \times 10^{-4}$ and

$3 \times 10^{-4}$. The range of the control parameter, however, is rather wide: $0<\epsilon \lesssim 1.5$. In 
this temperature range, the spectra have a single peak exactly at a half the FS frequency, and the SS attenuation length is less than the horizontal size of the cell. The typical 3D plot of the SS spectral density as a function of the FS amplitude and the frequency shift $\delta f=f-F / 2$ is shown in Fig.14.

The narrowness of the temperature range where good quality data are observed, can be explained by several reasons. The ratio $l / L$ in this temperature range changes from 0.55 to 0.95 . At $\tau<2 \times 10^{-4}$ the SS attenuation length becomes comparable with the distance between the FS transducers $(d=3.9 \mathrm{~mm})$. Therefore the SS waves that reach the bolometers, are generated near the cell edge where a FS acoustic field is inhomogeneous. In this region, we observed qualitatively the same SS spectra shifted a few Hertz to the right from the basic SS frequency.

At $\tau>3 \times 10^{-4}$ two other factors come into the play. The first one is the resonance cavity effects discussed in Sect.IB3 and IVA. It causes the transition from one to two peaks spectrum. The second, as the ratio $l / L$ exceeds unity, the lateral boundary conditions become important.

At the threshold of the parametric instability, the SS wave spectrum is very narrow and it starts widening as the control parameter increases. However, in the entire experimental range of the control parameter, the SS spectrum remains very narrow compared to other characteristic frequency scales. The smallest among them is the SS attenuation rate, $\gamma_{2}$, that is about $40-60 \mathrm{~Hz}$. The typical SS spectra for different values of the control parameters are shown in Fig.15. They have a finite width, $\Delta$, and exponentially decaying tails. In semilogarithmic coordinates the spectra look like triangles with equal sides. The width of the spectrum grows as the control parameter increases. In order to analyze the dependence of the spectral width on the control parameter and temperature, we fit the data by one of simple functions which possess the exponential tails, namely

$$
N(f-F / 2)=\cosh ^{-1}\left(\frac{f-F / 2}{\Delta}\right) .
$$

We emphasize here that the characteristic width of the wave packet, $\Delta$, is 20-50 times smaller 
than $\gamma_{2}$ and is the result of the nonlinear indirect $4 \mathrm{~W}$ interactions [22, as explained in the Sect. IIC2.

Fig. 16 presents the dependence of the spectral width as a function of the FS amplitude for different temperatures. At each temperature, the initial parts of the curves show a rather weak dependence on the control parameter. Then in a wide range of the control parameter $0.1<\epsilon<1.5$, the spectra width is fitted rather well by a linear function of the FS amplitude: $\Delta=m\left(A / A_{0}-1\right) . A_{0}$ differs from $A_{t h}$ due to flat initial part of the curves, as it is seen from the plot. The temperature dependence of the slope $m$ is shown in the inset of Fig.16. It increases about twice in the narrow temperature range very similar to the SS attenuation rate, $\gamma_{2}$. A comparison of the experimental, almost linear dependence of the spectral width on the control parameter with the theoretical predictions for different space dimensionalities of the SS waves (see Eq.(25)), allows us to conclude that the experimental situation is either 1D or lies between 1D and 2D cases. The temperature change of the prefactor in Eq.(25) is also close to the experimentally observed temperature dependence of the coefficient $m$.

\section{Statistical analysis of the SS amplitudes.}

In the same temperature range, where the SS spectra broadening was measured, we also conducted the statistical analysis of the SS amplitude signals measured by both bolometers (see Fig.13). We intended to answer the question whether the statistics of the SS wave amplitudes was Gaussian. The characteristic plots of the SS amplitude time series are shown in Fig.13.

One of the parameters that characterizes the deviation of the amplitude distribution function from the Gaussian one, is the ratio: $\Phi=M_{4} / M_{2}^{2}$, where $M_{2}$ and $M_{4}$ are the second and the fourth order statistical moments: $M_{2}=\left\langle(Z-\langle Z\rangle)(Z-\langle Z\rangle)^{\star}\right\rangle$ and $M_{4}=$ $\left\langle(Z-\langle Z\rangle)^{2}(Z-\langle Z\rangle)^{\star 2}\right\rangle, Z$ is the complex wave amplitude, $\langle\ldots\rangle$ means averaging over time series.

A characteristic plot of $\Phi$ as a function of the FS amplitude at a fixed temperature is 
shown in Fig.17. Below the threshold $\Phi=2$, that provides an evidence of the Gaussian distribution of complex amplitudes of an experimental noise without any phase correlation. Near and above the threshold $\Phi$ fluctuates strongly. At $\epsilon>1 / 2$ it approaches an average value of about 3 .

The statistics is Gaussian if the fourth order correlation function is equal to the sum of all possible products of the pair correlation functions:

$$
\left\langle b_{1} b_{2} b_{3}^{*} b_{4}^{*}\right\rangle=\left\langle b_{1} b_{3}^{*}\right\rangle\left\langle b_{2} b_{4}^{*}\right\rangle+\left\langle b_{1} b_{4}^{*}\right\rangle\left\langle b_{2} b_{3}^{*}\right\rangle+\left\langle b_{1} b_{2}\right\rangle\left\langle b_{3}^{*} b_{4}^{*}\right\rangle
$$

where $b_{i}=b\left(\mathbf{k}_{\mathbf{1}}, \omega_{1}\right)$ is the SS wave amplitude for given $\mathbf{k}_{\mathbf{i}}$ and $\omega_{i}$. Two first terms are products of the second order normal correlators:

$$
\left\langle b_{\mathbf{k}_{1}, \omega_{1}} b_{\mathbf{k}_{2}, \omega_{2}}^{*}\right\rangle=n_{\mathbf{k}_{1}, \omega_{1}} \delta\left(\mathbf{k}_{1}-\mathbf{k}_{2}\right) \delta\left(\omega_{1}-\omega_{2}\right)
$$

$n_{\mathbf{k}_{1}, \omega_{1}}$ is the number of waves with given $\mathbf{k}_{\mathbf{i}}$ and $\omega_{i}$, or the spectral wave density. The third term is a product of the anomalous correlators:

$$
\left\langle b_{\mathbf{k}_{1}, \omega_{1}} b_{\mathbf{k}_{2}, \omega_{2}}\right\rangle=\sigma_{\mathbf{k}_{1}, \omega_{1}} \delta\left(\mathbf{k}_{1}+\mathbf{k}_{2}\right) \delta\left(\omega_{1}-\omega_{2}\right)
$$

The anomalous correlator differs from zero for parametrically generated waves because a pumping field produces pairs of waves, and the waves inside one pair are strongly correlated. It was shown theoretically [10], that in a steady state $\left|\sigma_{\mathbf{k}, \omega}\right|=\left|n_{\mathbf{k}, \omega}\right|$, and that leads to the following relation between statistical moments:

$$
M_{4}=3 M_{2}^{2}
$$

The relation between the fourth and second moments with zero anomalous correlator is different: $M_{4}=2 M_{2}^{2}$. Below the threshold of the parametric instability the measured signal is the electronic noise. This noise certainly has no anomalous correlator, and it is Gaussian, i.e., $\Phi=2$. Close to the threshold the value of $\Phi$ fluctuates strongly. One of the reasons for such fluctuations is not sufficient time of the statistical measurements. The characteristic time scale of the amplitude fluctuations for small $\epsilon$ is comparable with the measuring time 
(see Fig.13). For large $\epsilon, \Phi$ becomes equal to 3, and this is a crucial evidence of the Gaussian statistics of the SS wave amplitudes. This transition in the value of $\Phi$ from 2 below the threshold to 3 above it, indicates a very crucial manifestation of a physical mechanism for the parametric instability, namely, appearance of a parametrically initiated wave pairing with a strong phase correlation inside of each wave pair.

We verified the Gaussian statistics only for the correlators with a time delay and a space shift both equal zero. So we got necessary but not a sufficient proof of the Gaussian statistics. However, it is the first experimental evidence of the validity of the theoretical hypothesis about the Gaussian statistics for the parametrically generated waves.

The Gaussian statistics of the SS amplitude fluctuations above the threshold verified in the experiment, unambiguously points at the kinetic theory to describe the behavior of the parametrically excited SS waves and provides a foundation for its applicability. In this approach, one introduces pair correlation functions of parametrically excited waves and, using a hypothesis of the Gaussian statistics of wave amplitudes, splits higher order correlation functions, that appear in nonlinear terms, into products of the pair correlation functions [10]. The kinetic theory also predicts that a spectral packet has universal exponential tails and its width is small due to relatively weak $4 \mathrm{~W}$ scattering processes. Therefore, the observed shape and width of the packet confirm the main results of the kinetic theory.

We also measured the crosscorrelation function of the amplitude signals, $Z_{1}$ and $Z_{2}$, from two bolometers in the first cell:

$$
C(\theta)=\frac{\left\langle\left(Z_{1}(t)-\left\langle Z_{1}\right\rangle\right)\left(Z_{2}(t-\theta)-\left\langle Z_{2}\right\rangle\right)^{\star}\right\rangle}{\sqrt{\left\langle\left|Z_{1}(t)-\left\langle Z_{1}\right\rangle\right|^{2}\right\rangle \cdot\left\langle\left|Z_{2}(t)-\left\langle Z_{2}\right\rangle\right|^{2}\right\rangle}}
$$

The bolometers are sensitive to the waves propagating in almost perpendicular directions. The absolute value of the normalized crosscorrelation function is found to be $|C(\theta)|<0.1$ for all delays $\theta$. That can be explained by an absence of angular correlations between the SS waves, and once more indicates a random phase distribution of wave amplitudes already discussed above. Moreover, we were able to measure correlations of the SS signals obtained by the different bolometers also in the second cell. We found no significant correlations 
between the signals on the bolometers near the threshold instability. That indicates no tendency to pattern formation. This observation agrees with the theoretical conclusion about the absence of a long-range order at an angle of resonantly interacting SS waves. As we suggested above (see Sect.[एC1), this state, particularly very close to the instability threshold, can be described as a turbulent crystal [35].

The $4 \mathrm{~W}$ resonance interaction is present in any medium with parametrically driven waves. Thus the kinetic theory predicts the universal spectral broadening in such systems irrespective of the wave dispersion law. We do not exclude a possibility that this universal spectral broadening can be observed in the capillary waves too. This would be possible if the resolution in frequency measurements is drastically improved compared to that reached in previous experiments, to be much better than the ratio $\gamma / \omega$, which for the Faraday ripples is of the order $10^{-2}$.

\section{Experiment on simultaneous FS and SS pumping}

\section{Acoustic phase conjugation in superfluid helium.}

The experiments in the second cell with a simultaneous FS and SS pumping reveal a new effect in the dynamics of the SS waves below the parametric instability threshold. An analog of the well-known in optics phenomenon of a phase conjugated (PC) mirror was observed in the superfluid helium for the SS waves. A mirror built on this effect, in a contrast to a conventional one, reflects an incident wave in such a way that a reflected wave is always directed opposite to an incident one.

One of the differences between the first and the second cells essential for such experiments is the presence of the bolometer located between the SS emitter and the cell. Thus, in the second cell, we had an opportunity to measure the SS wave amplitude emitted from the heater before it entered into the cell. On the same bolometer we could measure an amplitude

of the SS wave that was coming back from the cell. Another great advantage of the second 
cell, which allows to perform the experiments on the phase conjugation, is a wide angle sensitivity of the fiber bolometers and absence of a reflection for the SS waves.

\section{Theoretical background.}

A PC mirror has several unique properties compared to an ordinary mirror. It reflects an incident wave back for any incident angle. The conjugated wave can have a larger amplitude than the incident one. But it is this time-reversed phase property of the reflected wave that makes the optical PC so potentially useful for a host of interesting applications, and particularly for correction of wavefront distortions [53]. A common but not a sole realization of PC in optics is the mirror, based on $4 \mathrm{~W}$ interactions [53]. It can also be realized through $3 \mathrm{~W}$ interactions. In the latter case, $\mathrm{PC}$ has been observed in various wave systems manifesting sufficiently strong nonlinear interactions, e.g. microwaves [54 and acoustic waves [55]. In fact, the first observation of $\mathrm{PC}$ in acoustics was made long before the observation of the optical PC [55. PC in acoustics results from the interaction between sound waves and the various types of collective oscillations in solids. The interaction of a sound wave (either longitudinal or shear) with electromagnetic waves in piezoelectrics or magnets, the phononplasmon interaction in piezoelectric semiconductors, and the interaction of electromagnetic waves with spin waves in magnets are a few of many examples. At large enough amplitudes of an external field, these systems exhibit a space-homogeneous parametric instability, as was already described above.

The PC phenomenon of the SS waves in the superfluid helium can be observed below as well as above the parametric instability threshold.

An incident SS wave with a half the frequency of the FS wave can be amplified by the FS pumping wave, generating a PC wave in the opposite direction. Indeed, as can be easily seen from the conservation laws of Eq.(5), at $|\mathbf{K}| \ll\left|\mathbf{k}_{\mathbf{1 , 2}}\right|$ one gets $\omega_{1,2} \approx \Omega / 2$, and the parametrically generated SS waves propagate in almost opposite directions $\mathbf{k}_{\mathbf{2}} \simeq-\mathbf{k}_{\mathbf{1}}$ with the conjugated phases.

Two main factors distinguish our system from the common manifestation of PC in optics. First, PC in optics is usually examined only below the onset of spontaneous oscillations 
(instability), which is unattainable at the currently available laser intensity [53]. Second, the optical systems are of a very low dissipation, so that $l / L \gg 1$. Our system is in the range of $l / L \geq 1$ depending on the temperature, so the dissipation is of a crucial importance for the conjugated wave generation.

Two linear (in respect to the incident and conjugated waves amplitudes) problems can be formulated in regards to the parametric instability of FS: 1) determination of the threshold of a spontaneous SS wave generation via a $3 \mathrm{~W}$ interaction process, 2) generation of the conjugated SS wave below the instability onset due to the nonlinear interaction of the incident SS wave with the FS pumping field.

The first problem, discussed in Sect.IIA2 and IVA, deals with the spatially uniform, rotationally invariant state in a FS resonance cavity.

The second problem is related to a system with its rotational invariance externally broken by the incident SS wave having a spatially dependent amplitude. The relevant question here is: what is the amplitude value of the conjugated SS wave which is generated as a result of the $3 \mathrm{~W}$ interaction? Depending on the boundary conditions and the value $l / L$ for the same cell geometry this problem can be described in two ways. First method is similar to the PC paradigm in optics [53]. One can consider the inhomogeneous problem for a spacedependent amplitude distribution of the probe and the conjugated waves in a resonance cavity with a dissipation. In this case the nonlinear process of the PC wave generation via the $3 \mathrm{~W}$ interactions (with non-zero wave number mismatch $\Delta k=\delta / c_{2}$ ) is described by the following set of linear steady state equations

$$
\begin{aligned}
& \left(\partial / \partial x+\gamma_{2} / c_{2}\right) b_{1}+i \kappa b_{2}^{*} \exp (i \Delta k x)=0 \\
& \left(\partial / \partial x+\gamma_{2} / c_{2}\right) b_{2}+i \kappa b_{1}^{*} \exp (i \Delta k x)=0
\end{aligned}
$$

and the boundary conditions for both incident and conjugated waves:

$$
b_{1}(0)=b_{0}, \quad b_{2}(L)=0
$$

Here $\kappa \equiv a U / c_{2}, b_{1,2}$ are the incident and conjugated SS wave amplitudes, respectively, $0 \leq x \leq L$ is the coordinate in the direction of the SS wave path, and $b_{1,2}(0)$ are the 
amplitudes of the incident and the reflected SS waves at the cell entrance, respectively. Rather straightforward calculations lead to the following expression for the nonlinear power reflection coefficient, defined as $r=\left|b_{2}(0)\right|^{2} /\left|b_{1}(0)\right|^{2}$ :

$$
r=4 \kappa^{2} \frac{\cosh (2 g L)-\cos (2 f L)}{D},
$$

where

$$
\begin{aligned}
D & =\exp (-2 g L)\left[(f-\Delta)^{2}+\left(g-\gamma_{2} / c_{2}\right)^{2}\right]+ \\
& +\exp (2 g L)\left[(f+\Delta)^{2}+\left(g+\gamma_{2} / c_{2}\right)^{2}\right]+ \\
& +4\left(g^{2}-\left(\gamma_{2} / c_{2}\right)^{2}\right) \cos (2 f L)+4\left(f \gamma_{2} / c_{2}-g \Delta\right) \sin (2 f L), \\
f^{2}-g^{2} & =\kappa^{2}+\Delta^{2}-\left(\gamma_{2} / c_{2}\right)^{2}, \quad f g=\Delta \gamma_{2} / c_{2} .
\end{aligned}
$$

Eqs.(3840) are transformed to the known expression for the power reflection coefficient in the optical PC via a nearly-degenerate $4 \mathrm{~W}$ mixing at $\gamma \rightarrow 0$ [53]. At this limit, in the region $\pi / 4<\kappa L<\pi / 2$, the intensity of the PC wave exceeds that of the incident one, i.e., it is the regime of the $\mathrm{PC}$ coherent amplifying reflector. The dissipation cuts down the amplification and shifts the amplification region closer to the onset of the parametric instability. Thus both the dissipation and the frequency mismatch result in a decrease of the PC reflectivity, and the conjugator behaves as a narrow bandpass acoustic filter. In the limit $a / a_{t h} \ll 1$, Eq.(38-40) can be simplified

$$
r=4 \kappa^{2} \frac{\cosh (2 g L)-\cos (2 f L)}{\exp \left(2 L \gamma_{2} / c_{2}\right)\left[\left(\delta / c_{2}\right)^{2}+\left(\gamma_{2} / c_{2}\right)^{2}\right]} .
$$

This limit can be always achieved, and the expression (41) can be easily analyzed. Indeed, it follows that: (i) a reflected wave amplitude, $\left|b_{2}(0)\right|$, is proportional to a FS amplitude, $a$; (ii) $\left|b_{2}(0)\right|$ is proportional to an incident wave amplitude, $\left|b_{1}(0)\right|$; (iii) a reflection coefficient has a resonant dependence on frequency mismatch, $\delta$. The resonance curve depends on the SS attenuation rate, $\gamma_{2}$, and the cell size, $L$.

However, as our calculations and comparison with the experiments show, the expressions either of Eqs.(38-40) or Eq.(41) do not fit the experimental data. This is, probably, due to the relatively large dissipation and, particularly, due to the nonreflecting lateral boundaries. 
The second method is to consider a homogeneous problem for the probe and the conjugate waves with a linear decay. Thus, in this case the nonlinear process of the PC wave generation via the $3 \mathrm{~W}$ interaction is described by the following set of linear equations:

$$
\begin{gathered}
{\left[\partial / \partial t+\gamma_{2}+i \omega_{1}\right] b_{1}+i U a b_{2}^{*}=0} \\
{\left[\partial / \partial t+\gamma_{2}-i \omega_{2}\right] b_{2}^{*}-i U^{*} a^{*} b_{1}=0}
\end{gathered}
$$

Here, the incident and the conjugated SS waves have the amplitudes $b_{1}$ and $b_{2}$ and the frequencies $\omega_{1}$ and $\omega_{2}$, respectively, and $\omega_{1,2}=\Omega / 2 \pm \delta$, where $\delta$ is the frequency shift of the parametrically generated SS waves. The nonlinear power reflection coefficient is obtained from eqs. (42-43) as:

$$
r=\frac{|U a|^{2}}{\gamma_{2}^{2}+\delta^{2}} .
$$

Here $b_{1,2}(0)$ are the amplitudes of the incident and the reflected SS waves, respectively, at the cell entrance.

In order to describe the experimental data by Eq.(44), obtained in a finite lateral geometry FS resonance cavity, one can rewrite Eq.(44) by incorporating Eq.(16) in the following form:

$$
r=\left(\frac{a}{a_{t h}}\right)^{2} \frac{\zeta^{2}\left[1+(\xi l / L)^{2}\right]}{1+\left(\delta / \gamma_{2}\right)^{2}} .
$$

This approach is justified in the linear regime below the parametric instability threshold. The effective attenuation $\Gamma=\gamma_{2}-\sqrt{|U a|^{2}-\delta^{2}}$ tends to zero at the threshold and becomes negative above it, causing the SS wave amplitude to diverge exponentially. Saturation occurs due to higher-order nonlinear effects of the SS wave interaction (see Sect.[IC1 and IVB).

The main theoretical predictions for the PC waves generated parametrically below the onset are:

1. The amplitude of the conjugated SS wave is proportional to: (a) the FS amplitude at fixed value of the frequency shift, and (b) the amplitude of the incident SS wave.

2. The sum of the phases of one FS and two SS waves involved in the resonance interaction, 
is $\phi+\phi_{1}+\phi_{2}=\pi / 2$. For a pair of the SS waves this leads to $\phi_{1}+\phi_{2}=$ const.

3. The power reflection coefficient as a function of the frequency shift has a Lorentzian shape with a width equal to the SS wave linear attenuation rate.

Results and discussion.

As in the previous experiments, we performed the SS spectrum measurements in a narrow bandwidth around $\Omega / 2$ using the lock-in amplifier fixed at this reference frequency (see Sect.[IIF 1). The experiment was conducted in the second cell at a fixed temperature by pumping the acoustic cavity with the FS waves at the frequency $F$ and the amplitude below the threshold value for the parametric instability. At the same time, a small amplitude SS wave was emitted by the heater. In order to separate SS signals coming directly from the heater and those coming from the cell, the incident wave frequency was shifted from $F / 2$ to $f_{1}=F / 2+\delta f$.

A bolometer B8, located far from the heater (see Fig.5), detected only the SS waves with the frequency $f_{1}$. A bolometer B4, on the other hand, located between the heater and the cell, detected two signals: one with the frequency $f_{1}$ coming from the heater, and another with the frequency $f_{2}=F / 2-\delta f$ coming from the cell. This dual detection is clearly seen from the power spectrum of the signal read by the bolometer B4 (see inset of Fig.18). The spectra were measured at the reduced temperature $\tau=7.07 \times 10^{-4}$ and the FS amplitude $A=75 \mathrm{~Pa}$. This amplitude was below the threshold value $A_{t h}=162 \mathrm{~Pa}$, measured at the same temperature. The dependence of the amplitudes of both peaks at the same temperature on the FS amplitude is presented in Fig.18, the frequency shift is $\delta f=-12 \mathrm{~Hz}$. The amplitude of the incident SS wave $b_{1}$, arriving directly from the heater on the bolometer B4, clearly does not depend on the FS amplitude, since these waves do not interact in the region between the heater and the resonance cavity. On the other hand, the amplitude of the SS waves arriving from the cell, $b_{2}$, is a linear function of the FS amplitude below the parametric instability threshold, as follows from the theory, (see Eq.(44)), and is also a linear function of the SS wave amplitude emitted by the heater $b_{1}$. The estimate of the slope of $b_{2} / b_{1}$ as a function of $A / A_{t h}$ from Fig.18 gives $1.7 \pm 0.15$. This value is in a fair 
agreement with the theoretical value of 2.05 obtained from Eq.(44). Thus, the first property of the conjugated SS signal, following from Eq.(44), is quantitatively verified.

Particular efforts were made to verify the phase relation between the incident and the conjugated SS waves. The phases of each of the SS signals at the frequencies $f_{1}$ and $f_{2}$ were arbitrary with respect to the pumping field, and changed randomly from one measurement set to another (open circles in Fig.19). However, the sum of the two phases of both signals was constant for all FS and SS incident wave amplitudes (Fig.19). The standard deviation from the average value of the phase sum was $\delta\left(\phi_{1}+\phi_{2}\right)=0.15 \mathrm{rad}$, as compared to the uniform distribution of the phase, in a $2 \pi$ bandwidth, for each separate signal. This result is the main evidence for the $\mathrm{PC}$ in the $\mathrm{SS}$ waves.

The dependence of the nonlinear reflection coefficient, $r$, on the frequency shift, $\delta f$, at the reduced temperature $\tau=7.07 \times 10^{-4}$ and the FS amplitude $A=75 \mathrm{~Pa}$ is presented in Fig.20. Fitting the experimental data by the Lorentzian function gives a bandwidth of $\Delta=14 \mathrm{~Hz}$ compared with the theoretical width $\Delta_{t h}=19 \mathrm{~Hz}$. The theoretical width is defined solely by the SS attenuation in an infinite cell at the experimental values of $\tau$ and the SS frequency $F / 2 \approx 20 \mathrm{kHz}$. The main reason for the discrepancy is a near-field configuration, used in the experiment. The latter can lead to distortions of the linear decay rate.

In conclusion, we have presented the first direct evidence of PC in the SS waves, as a result of the $3 \mathrm{~W}$-interaction between the FS pumping field and the SS waves, below the threshold of the parametric instability. Since PC is a fairly general phenomenon in parametrically generated waves, we are convinced that it can be observed in other parametrically driven systems. One of these easily-accessible systems is that of the surface waves parametrically excited by a vertical vibration [11]. An obvious advantage of the latter system is an easy visualization of the surface waves which can be used to verify the PC effect experimentally. 


\section{Experiments on simultaneous FS and SS pumping above the threshold.}

One of the powerful tools to probe a state of many waves is to measure its response on small perturbations, namely on an externally excited, small amplitude wave. An independent SS wave emitted from a heater, propagates through the nonlinear medium of the interacting waves, and interacts with them. We study experimentally the response of the parametrically generated SS waves above the threshold on a small perturbation in both cells.

Amplification of externally generated SS waves by FS above the threshold.

The first experiment on an amplification of the SS waves by FS was conducted in the first cell. The small amplitude SS wave was emitted from the plane heater (see Fig.3) at exactly a half the FS frequency, $F / 2$. The signal was measured on the bolometer located on the opposite side of the cell. The SS intensity was measured as a function of the FS amplitude for the different initial SS amplitudes and the different reduced temperatures, $\tau$.

The amplitude of the emitted wave was calculated by using the data for the SS wave attenuation [19] and the measured SS wave amplitude on the bolometer. The typical plots of the SS intensity, $I$, as a function of the FS amplitude, $A$, for different values of a heat flux from the heater, i.e., the different SS amplitudes of the probe wave, are shown at $\tau=3.32 \times 10^{-4}$ in Fig.21. Each plot can be approximated by two linear regions: the first region for sufficiently small FS amplitudes below the instability onset, and second one is above the threshold.

For sufficiently small FS amplitudes, the linear attenuation and the 3W interactions between the FS and SS waves define the SS amplitude. Below the threshold of the parametric excitation the effective attenuation rate is

$$
\Gamma=\gamma_{2}-a U
$$

It can be rewritten as $\Gamma=\gamma_{2}\left(1-a / a_{t h}^{\infty}\right)$. Then the SS wave intensity on the bolometer far below the threshold can be expressed as

$$
I=I_{0}^{\prime}\left(\frac{A}{A_{t h}}+1\right), \quad I_{0}^{\prime}=I_{0} \exp \left(-\frac{2 L \gamma_{2}}{c_{2}}\right),
$$


where $I_{0}^{\prime}$ is the SS wave intensity, reaching the bolometer without FS pumping. Using Eq.(47) to fit the initial part of plots in Fig.21, one gets the threshold FS amplitudes and the SS wave intensity on the heater, $I_{0}$.

The SS wave amplitude above the threshold is defined by the nonlinear attenuation, discussed in Sect. ПC1 and एVB. Similar to the results on the SS intensity, discussed there, the SS intensity slope, $g_{\text {exp }}$, was found to be much smaller than the theoretically predicted one [22].

However, a new unexpected giant amplification of the SS probing waves by the FS pumping was observed in the first cell. The SS intensity as a function of the control parameter, $\epsilon$, on the plots with different $I_{0}$ and for different $\tau$ was fitted by $I_{2}=g_{\exp }\left(I_{0}, \tau\right) \epsilon$. The threshold, $A_{t h}$, was found to be almost independent on the initial SS intensity, $I_{0}$, and was the same as measured in the previous experiments without a simultaneous SS pumping (see Sect.IVA). As it was discussed before, the SS intensity above the threshold fluctuates strongly in the first cell without a simultaneous SS pumping (see Sect.IVB2). With the simultaneous SS pumping at a sufficiently small SS amplitude, the intensity of the measured signal still remains fluctuating. As the SS pumping amplitude increases the fluctuations are suppressed, and the slope $g_{\text {exp }}$ strongly increases. Although, the maximum value of the slope is still remained lower than the theoretically predicted value [22]. Plots of the dependence of the SS intensity slope as a function of the SS probe wave intensity for the different reduced temperatures are presented in Fig.22.

Very close to $T_{\lambda}$, the SS intensity slope strongly depends on the initial SS pumping intensity. The value of $g_{\text {exp }}$ changes few orders of magnitude as $I_{0}$ increases. This effect becomes weaker as $\tau$ increases. The data $g_{\text {exp }}\left(I_{0}\right)$ for various $\tau$ is plotted in the logarithmic scale in Fig.22. These experimental data can be fitted by a function $g \sim I_{0}^{\nu}$, where the index $\nu$ in its turn is a function of the reduced temperature. The inset in the Fig.22 shows the dependence $\nu(\tau)$. This dependence can be fitted by $\nu=\tau_{0} / \tau-\nu_{0}$. The fit parameters were found to be : $\tau_{0}=5.2 \times 10^{-4}$ and $\nu_{0}=1$. So, finally one has $\nu=\tau_{0} / \tau-1$. This data representation has no theoretical background whatsoever. However, it allows to make some 
speculations about possible reasons for the giant amplification of the SS probe waves.

As we already discussed above (see Sect.[VB 1), one of the possible explanations of huge quantitative discrepancy between the theoretically predicted value for $g$ and the experimental ones for $g_{\text {exp }}$, measured without the SS simultaneous pumping, may be the nonuniform spatial structure of a SS wave packet that was not taken into account by the theory [22]. For the sufficiently small $\tau$, the SS wave spectra exhibit only one central peak (see Sect. IVA). Then the SS waves propagate almost in the cell plane. However, a SS wave packet has nonuniform amplitude distribution in the vertical direction. As was discussed above, the most preferable conditions for the SS wave generation are in narrow layers near the FS transducers, because the amplitude of the pressure oscillations has a maximum there. On the other hand, the SS bolometers in the first cell are located in the cell midplane and thus have low sensitivity to the waves propagating close the FS transducer surfaces. Moreover, two SS waves, propagating along two transducer surfaces maybe almost incoherent and have a random destructive interference on the bolometers. All these factors could drastically reduce the measured SS intensity, as we already discussed in Sect.IVB 1.

In the experiment with the simultaneous FS and SS pumping the plane SS wave, emitted by the heater, had a uniform amplitude distribution in the vertical direction. Such a wave interacted with the FS wave field and could lead to a redistribution of a SS packet intensity at the bolometer. The emitted SS wave can also synchronize phases of two packets propagating along two FS transducers and suppress the fluctuations of interference signal. These factors alone can increase greatly the SS wave intensity measured on the bolometer. This effect should be certainly sensitive to initial SS wave distribution and, thus, to the reduced temperature.

At large $\tau$, the SS wave spectra became splitted, and one wave in a pair of the SS waves propagates out of the cell plane. So the emitted SS wave from the heater may not have such strong influence on the SS wave intensity redistribution. The reduced temperature at which the influence of the SS pumping wave on the intensity slope $g_{\text {exp }}$ vanishes $\left(\tau_{0}=5.2 \times 10^{-4}\right.$, found from the fit of the data in Fig.22 ) lies surprisingly close to the value of $\tau$, at which 
the transition from one peak to the splitted type of SS spectrum occurs. The SS reflection from the lateral boundaries becomes important, and discrete levels come into the play. At $\tau>\tau_{0}$ the slope $g_{\text {exp }}$ does not change with $I_{0}$ variations. In this temperature range, another effect which modifies the SS intensity measurements, takes over.

Nonlinear SS wave resonances in the first cell with lateral reflection.

A typical plot of the SS wave intensity as a function of the FS amplitude for a given SS pumping amplitude at $\tau=5.21 \times 10^{-4}$ is shown in Fig.23. As seen clearly from the plot, the SS intensity strongly oscillates as the FS amplitude changes. The most surprising fact is that for some values of the control parameter the SS wave intensity reaches zero values. One of the reasonable explanations can be the effect of nonlinear renormalization of the SS wave number due to the $3 \mathrm{~W}$ interactions [10]. In all nonlinear wave systems, the $3 \mathrm{~W}$ interactions renormalize the wave frequency. It means that the dispersion relation becomes dependent on the amplitude of the parametrically excited waves as

$$
\omega_{n l}=\omega(k)+2 \int T\left(k, k^{\prime}\right) b\left(k^{\prime}\right)^{2} d k^{\prime},
$$

where $\omega(k)$ is the linear dispersion relation, $T$ is the $3 \mathrm{~W}$ interaction matrix element, and $b\left(k^{\prime}\right)$ is the SS wave amplitude. In the case of the parametric wave generation, the pumping frequency, $\Omega$, is fixed, and frequency of the parametrically excited waves always satisfies the condition $\omega_{n l}=\Omega / 2$. Then the Eq.(48) leads to the renormalization of the wave vectors of the excited waves to satisfy the frequency relation. In the case of a cell opened in a lateral direction, such an effect does not influence on an average intensity of parametrically excited waves. However, in a resonance cavity it leads obviously to resonance oscillations of the wave intensity, detected by a bolometer. In the first cell at the reduced temperature larger than $\tau \approx 5 \times 10^{-4}$, the finite size effect became significant since the SS dissipation length became larger than the cell size, $l / L>1$ (see Sect.IVA 2). Then the SS reflection at the lateral boundaries could modify the dependence of the SS intensity on the FS amplitude. Thus, the probable explanation of the strong SS intensity oscillations as a function of the FS amplitude could be the nonlinear renormalization of the dispersion relation of the para- 
metrically generated SS waves and influence of the reflecting lateral boundaries on the SS measured intensity. We would like to emphasize here that a similar effect of the strong SS wave intensity oscillations but less pronounced, was observed in the first cell even without an additional SS pumping, as we already pointed out in the Sect. $\mathbb{I V A}$ and Fig.7.

Let's estimate the value of the control parameter variation, $\Delta \epsilon$, which is necessary to switch from one to another, close-by resonance SS modes. In the case of the SS parametrically driven waves, Eq.(48) can be written as 22

$$
\omega_{n l}=\omega+\operatorname{Re} \Sigma
$$

where $\operatorname{Re} \Sigma$ is the real part of the correction for the self-energy function $\Sigma$, calculated in Ref. [22]. For one-dimensional geometry the calculations give

$$
\Delta \omega \equiv \omega_{n l}-\omega=\frac{4 B^{2} b^{2}}{\omega} \ln \left(\frac{\omega^{2}}{320 \gamma_{2}^{2}}\right)
$$

where $b^{2}=I_{2} / c_{2} \omega$ and $B$ is defined in Eqs.(20-22).

By substituting $I_{2}$ and $g$ from Eqs.(17 and 18) one gets

$$
\frac{\Delta \omega}{\omega}=3.44\left(\frac{\gamma_{2}}{\omega}\right)^{2} \Delta \epsilon
$$

This nonlinear frequency shift causes a change in the nonlinear wave length: $\frac{\Delta \omega}{\omega}=\frac{\Delta \lambda}{\lambda}=$ $(L / \lambda+1)^{-1}$. Then the variation in the control parameter necessary for switching to a neighboring SS mode is

$$
\Delta \epsilon \approx \frac{1}{3.44} \frac{\lambda}{L}\left(\frac{\omega}{\gamma_{2}}\right)^{2} \approx 50 \gg 1
$$

Thus again, we obtain large quantitative discrepancy between the theoretical estimates, based on the $3 \mathrm{~W}$ interactions, and the experimental results. It is feasible that it has the same source as the discrepancy in the value of the nonlinear coefficient $g$ for the amplitude saturation, which also results from the $3 \mathrm{~W}$ interactions.

Another possible mechanism to explain the phenomenon is a redistribution of the SS intensity between different azimuthal modes due to increase of a nonlinear SS interaction 
as the FS pumping amplitude raises. The azimuthal Bessel modes of the SS waves have much smaller differences between higher order modes in the spectrum than the longitudinal modes. Then one can expect that the energy redistribution between the modes due to the nonlinear interactions can bring into the resonance different modes for different values of the FS wave amplitude. Unfortunately, one cannot quantitatively estimate this effect.

\section{CONCLUSIONS.}

We present the results of the experiments on the parametric generation of the SS wave by FS in the superfluid helium in two resonant cavities with the different lateral boundary conditions and the different angular sensitivity of the SS detectors. There are three main subjects which were studied:

(i) the temperature dependence of the parametric instability threshold and the SS spectra at the onset;

(ii) the mechanism of the wave amplitude saturation and the nonlinear properties of the SS waves above the threshold, their statistical and spectral characteristics;

(iii) the interaction between the independently pumped SS wave and FS: SS phase conjugation below the threshold, and the giant amplification of the parametrically generated SS waves above the threshold.

Comparison of the presented experimental results with the theory, reveals the following. The temperature dependence of the FS threshold amplitude for the onset of the parametric instability in a resonance cavity of a finite lateral size agrees well with the theoretical predictions (see Fig.8) without any fitting parameter. The theory quantitatively explains also the experimentally observed transition from a single line SS wave spectrum close to $T_{\lambda}$, to a spectral spitting further from $T_{\lambda}$. It results from lifting of a degeneracy in the SS wave generation process when the SS dissipation rate becomes smaller than the frequency difference between two discrete resonance modes in the SS wave spectrum in the resonance cavity. 
In regards to the nonlinear properties of the SS wave ensemble, quantitative comparison is less successful. The strong fluctuations of the SS amplitude in the first cell, probably due to the lateral boundary reflections do not permit to get definite conclusions about its functional dependence on the FS pumping amplitude. In contrast, the results from the second cell provide an evidence of the mechanism of the SS amplitude saturation based on the functional dependence of the SS wave intensity on the control parameter for the different reduced temperatures. The linear dependence of the SS wave intensity in a wide range of the control parameter points out on the nonlinear attenuation due to the $3 \mathrm{~W}$ resonance interactions of the SS waves as a main mechanism of the SS amplitude saturation in agreement with the theory. However, the value of the nonlinear attenuation (or saturated SS intensity) greatly disagrees with the theoretical predictions. The possible reason for the great discrepancy lies in the interplay between nonuniformity of the SS wave packet profile due to its generation by FS in the resonance cavity, destructive interference of the SS waves coming from different directions on the bolometer, and spatial inhomogeneity of the bolometer sensitivity.

Another manifestation of the nonlinear SS wave interactions is observation and quantitative measurements of the SS spectral broadening with the exponential tails above the instability threshold in the first cell. The functional dependence of the spectral width on the control parameter and its temperature dependence agree well with the predictions, based on the kinetic theory of the $4 \mathrm{~W}$ (second order $3 \mathrm{~W}$ ) resonance interactions. The SS wave system provides a unique opportunity to get the first experimental evidence of the Gaussian distribution of the amplitudes of the parametrically generated waves. This fact supplies a firm ground for an application of the kinetic theory with a random phase approximation to describe statistical behavior of the parametrically excited SS wave ensemble.

Interactions of the system of the FS and parametrically generated SS waves with the SS wave pumped externally, reveal new effects. Below the instability threshold, a SS wave, phase conjugated to an incident SS wave, is excited as a result of the $3 \mathrm{~W}$ interaction between the FS pumping field and the SS waves. Three main features of the phase conjugation, predicted 
theoretically, were experimentally verified: (i) the linear dependence of the amplitude of the conjugated wave on the amplitude of the pumping field and on the amplitude of the incident wave, (ii) the resonance dependence of the amplitude of the conjugated wave on the frequency of the incident wave, and (iii) the phase relation between the incident and conjugated waves. Above the instability threshold two strong nonlinear effects were observed: the giant amplification of the SS wave intensity closer to the onset, and the strong resonance oscillations of the SS wave intensity as a function of the FS amplitude further from the threshold. There is no currently quantitative description of these effects. The qualitative explanation based on the interplay between the (i) nonuniformity of the SS wave profile, (ii) incoherence and destructive interference of the SS waves on the bolometer, (iii) discrete spectra of SS azimuthal modes in a cell plane, and (iv) nonlinear SS wave interactions and coherent action of the SS pumping wave, that cause redistribution of the SS wave intensity between different modes.

Let's now come back to the problem of a possible observation of the fully developed wave turbulence in a parametrically excited wave ensemble. In our experiments we observed the first instability of the FS wave. A state of a large number of the strongly interacting SS waves occurs as a result of the parametric instability at $L / \lambda \gg 1$. The SS wave ensemble has the Gaussian distribution of the wave amplitudes that justifies its description by the kinetic equation with a random phase approximation. the $4 \mathrm{~W}$ interactions generate new modes only in the vicinity of the main peak that results in the spectral broadening. However, this process is not strong enough even far from the threshold to redistribute modes in the SS wave spectra in a sufficiently wide wave number range. We have not got any evidence of the processes that may lead to creation the fully developed wave turbulence with a wide frequency spectrum. However, we cannot exclude a possibility that the fully developed wave turbulence may be observed at much higher energy fluxes, that is indeed rather problematic to realize experimentally. Thus, the parametrically generated SS waves is not suitable system to study the fully developed wave turbulence contrary to the theoretical expectations [36 38]. 


\section{ACKNOWLEDGMENTS.}

We would like to thank V. Cherepanov who was a coauthor of our early publications (Refs. 12,13]) and taught us the kinetic theory of the parametric instability of waves. We

are grateful to A. Muratov whose theoretical guidance on later stages helped us in the interpretation of our results. We are also thankful to V. L'vov, G. Falkovich, and V. Lebedev for many enlightening discussions and helpful criticism.

This work was partially supported by the Minerva Center for Nonlinear Physics and Complex Systems and by the Israel Science Foundation Grant No. 92/96. 


\section{REFERENCES}

[1] I. M. Khalatnikov, Introduction to the Theory of Superfluidity (Benjamin, New York, 1965).

[2] J. Wilks, The Properties of Liquid and Solid Helium, (Clarendon Press, Oxford, 1967).

[3] L. Goldner, N. Mulders, and G. Ahlers, J. Low Temp. Phys. 93, 131 (1993).

[4] H. Davidowitz and V. Steinberg, Physica D 84, 635 (1995).

[5] A. Yu. Iznankin and L. P. Mezhov-Deglin, Sov. Phys. JETP 57, 801 (1983).

[6] N. I. Pushkina and R. V. Khokhlov, JETP Lett. 19, 348 (1974).

[7] V. L. Pokrovskii and I. M. Khalatnikov, Sov. Phys. JETP 44, 1036 (1976).

[8] I. M. Khalatnikov, private communication.

[9] S. Garrett et al., Phys. Rev. Lett. 41, 413 (1978).

[10] V. L'vov, Wave Turbulence under Parametric Excitation. (Applications to Magnetics) Springer-Verlag, Berlin, 1994.

[11] M. C. Cross and P. C. Hohenberg, Rev. Mod. Phys.65, 851-1112 (1993).

[12] D. Rinberg, V. Cherepanov, and V. Steinberg, Phys. Rev. Lett. 76, 2105 (1996).

[13] D. Rinberg, V. Cherepanov, and V. Steinberg, Phys. Rev. Lett. 78, 4383 (1997).

[14] D. Rinberg and V. Steinberg, Phys. Rev. Lett. 81, 5812 (1998).

[15] D. Rinberg, Ph.D. thesis, ( Weizmann Institute of Science, Rehovot, Israel), 1997.

[16] V. L. Pokrovskii and I. M. Khalatnikov, Sov. Phys. JETP Lett 23, 653 (1976).

[17] S. K. Nemirovskii, Sov. Phys. Usp. 33, 429 (1990).

[18] G. Ahlers, in The Physics of Liquid and Solid Helium: Part I, edited by 
K. H. Bennemann and J. B. Ketterson(John Wiley and Sons, Inc., New York, 1976).

[19] R. Mehrotra and G. Ahlers, Phys. Rev. B 30, 5116 (1984).

[20] V. S. L'vov and A. M. Rubenchik, Sov. Phys. JETP 45, 67 (1977).

[21] V. B. Cherepanov and A. N. Slavin, in High Frequency Processess in Magnetic Materials, edited by G. Srinivasan and A. N. Slavin (World Scientific, Singapore, 1995).

[22] A. Muratov, Sov. Phys. JETP 112, 1630 (1997).

[23] I. V. Krutsenko, V. S. L'vov, and G. A. Melkov, Sov. Phys. JETP 48, 561 (1978).

[24] B. Ya. Kotyuzhanskii, L. A. Prozorova and L. E. Svistov, Sov. Phys. JETP 59, 644 (1984).

[25] V.S. L'vov and V.B. Cherepanov, Sov. Phys. JETP 48, 822 (1978).

[26] V. E. Zakharov, V. S. L'vov, and G. Falkovich, Kolmogorov Spectra of Turbulence I, Springer-Verlag, Berlin, Heidelberg, 1992.

[27] J. Hammack and D. Henderson, Annu. Rev. Fluid Mech.25, 55 (1993).

[28] W. Zhang and J. Vinals, J. Fluid Mech. 336, 301 (1997).

[29] W. S. Edwards and S. Fauve, J. Fluid Mech. 278, 123 (1994).

[30] P. Chen and J. Vinals, Phys. Rev. E 60, 559 (1999).

[31] A. Kudrolli and J. P. Gollub, Physica D 97, 133 (1996).

[32] D. Binks and W. van de Water, Phys. Rev. Lett 78, 4043 (1997).

[33] A. Ezerskii et al. Sov. Phys. JETP 64, 1228 (1986).

[34] N. B. Tufillaro, R. Ramshankar, and J. P. Gollub, Phys. Rev. Lett. 62, 422 (1989).

[35] A. C. Newell and Y. Pomeau, J. Phys. A: Math. Gen. 26, L429 (1993). 
[36] V. L. Pokrovskii, Sov. Phys. JETP Lett. 53, 585 (1991).

[37] G. V. Kolmakov and V. L. Pokrovskii, Physica D 86, 456 (1995).

[38] G. V. Kolmakov, Physica D 86, 470 (1995).

[39] V. Steinberg and G. Ahlers, J. Low Temp. Phys. 53, 255 (1983).

[40] H. Davidowitz, Ph.D. thesis, (Weizmann Institute of Science, Rehovot, Israel), 1992.

[41] J. Maynard, Phys. Rev. B 14, 3868 (1976).

[42] J. Heiserman. Methods of Experimental Physics V.19 Acoustic Measurments in Superfluid Helium, chapter 8, pages 413-453. Academic Press, 1981.

[43] W. R. MacLean., J. Acoust. Soc. Am., 12, 140-146, (1940).

[44] L. E. Kinsler, A. R. Frey, A. B. Coppens, and J. V. Sanders. Fundamentals of Acoustics, John Wiley and Sons, III edition, 1982.

[45] S. L. Garrett. Ph.D. thesis (University of California, Los Angeles, CA 90024, USA), 1977.

[46] M. Barmatz and I. Rudnick, Phys. Rev. 170, 224 (1968).

[47] D. Rinberg and M. Rappaport, Cryogenics, to be published.

[48] M. v. Schwerdtner, W. Poppe, and D. W. Schmidt, Cryogenics 29, 132 (1989).

[49] H. Davidowitz, Rev. Sci. Instr. 67 , 236 (1996).

[50] Syntheziser / function generator HP3325B, Hewlett Packard.

[51] Dynotrac lock-in ampifier model 393. Ithaco, Inc.

[52] Synthezised function generator model DS345. Stanford Research Systems.

[53] Optical Phase Conjugation, Ed. R. A. Fisher, (Academic Press), 1983. 
[54] R. Shih et al., Phys. Rev. Lett. 65, 579 (1990).

[55] R. B. Thompson and S. F. Quate, J. Appl. Phys. 42, 907 (1971), and see for review, e.g., F. Bunkin et al., Sov. Phys. Usp. 29, 607 (1986). 


\section{FIGURES}

FIG. 1. The vector diagrams for three wave resonance interactions: a. Cherenkov process; b. parametric decay process.

FIG. 2. The vector diagram of wave interactions in a resonance cavity.

FIG. 3. The first cell schematic drawing.

FIG. 4. Drawing of the flat bolometer.

FIG. 5. Schematic drawing of the second cell. The inset: the fiber bolometer wiring arrangement.

FIG. 6. The electronic scheme of the experiment: B - bolometers $(1,2, . .8), \mathbf{H}$ - heater, $\mathbf{T}$ - FS transducer, $\mathbf{M}-a c-d c$ mixer, G1, G2 - generators, $\mathbf{V}$ - voltmeter.

FIG. 7. SS wave intensity vs FS wave amplitude at fixed temperatures: I) the first cell for the reduced temperatures $\tau=2.3 \times 10^{-4}, 6.52 \times 10^{-4}$, and $1.20 \times 10^{-3} ;$ II) the second cell for $\tau=3.67 \times 10^{-4}, 6.36 \times 10^{-4}$, and $8.06 \times 10^{-4}$.

FIG. 8. The temperature dependence of the FS threshold amplitude for the parametric instability for: I) the first cell, and II) the second cell. Solid circles with error bars are experimental points; curves A-theory for an infinite cell without the spectral spiltting $(r=1, \zeta=1 / 2)$, curves B-theory for a finite cell with no reflection from the boundaries and with the SS spectral splitting ( $r=0, \zeta=1 / \sqrt{2}$ ); curves $\mathbf{C}$ (plot $\mathbf{I}$ )-theory for a finite cell with the reflection coefficient $r=0.7$ and with the SS spectral splitting $(\zeta=1 / \sqrt{2})$; curve $\mathbf{D}$ (plot II)-theory of finite cell without reflections $(r=0)$ and without the SS spectral splitting $(\zeta=1 / 2)$. Dashed lines separate the regions where different conditions $l / L<>1$ and $\Omega \eta^{2} / \gamma_{2}<>1$ are satisfied. 
FIG. 9. The SS frequency shift as a function of the reduced temperature, $\tau$. The insets show two types of the SS power spectrum in two temperature ranges: (a) $\tau=2.19 \times 10^{-4}$, (b) $\tau=6.52 \times 10^{-4}$.

FIG. 10. SS spectrum peak shift relatively to $F / 2$ vs the reduced temperature, $\tau$, just above the threshold of the parametric instability for different bolometers in the second cell.

FIG. 11. The SS intensity vs FS amplitude in the second cell, measured on the bolometer 4 at $\tau=3.3 \times 10^{-4}$. The dashed line is the linear fit of the intensity data above the threshold $A_{t h}$.

FIG. 12. Temperature dependence of the experimental slope coefficient, $g_{\text {exp }}$, for two bolometers 4 and 8 , in the second cell.

FIG. 13. SS amplitude fluctuations as a function of time for different values of $\epsilon=A / A_{t h}-1$, at $\tau=2.33 \times 10^{-4}$.

FIG. 14. 3D plot of SS power spectral density as a function of FS amplitude and SS frequency shift $\delta f=f-\Omega / 2$, at $\tau=2.33 \times 10^{-4}$.

FIG. 15. SS power spectra for different values of $\epsilon=A / A_{t h}-1$, at $\tau=2.33 \times 10^{-4}$.

FIG. 16. Width of the SS power spectra as a function of the FS amplitude for different temperatures. The inset: temperature dependence of the slope, $m$.

FIG. 17. Ratio $\Phi=M_{4} / M_{2}^{2}$ for the SS amplitude fluctuations as a function of FS amplitude at $\tau=2.33 \times 10^{-4}$.

FIG. 18. Amplitudes of incident, $a_{1}$, (open circles) and conjugated, $a_{2}$, (full circles) SS waves as a function of FS amplitude $A$ at $\tau=7.07 \times 10^{-4}$ and frequency shift $\delta f=-12 \mathrm{~Hz}$. The inset: The spectrum of the SS signal measured on the bolometer B4 at FS amplitude $A=75 \mathrm{~Pa}$. 
FIG. 19. Dependence of the phase of the conjugated SS waves, $\phi_{2}$, on FS amplitude (open circles), and dependence of the sum of phases, $\left(\phi_{1}+\phi_{2}\right)$, of the incident and conjugate SS waves on the FS amplitude(solid circles).

FIG. 20. Frequency dependence of the nonlinear reflection coefficient at constant FS amplitude $A=75 \mathrm{~Pa}$ and $\tau=7.07 \times 10^{-4}$. The dashed line is a fit of the experimental data by a Lorentzian curve with a bandwidth of $\Delta=14 \mathrm{~Hz}$.

FIG. 21. SS wave intensity vs FS amplitude measured by the bolometer located opposite to the heater that emitted SS wave, in the first cell, for different heating power $w_{h}$ at $\tau=3.32 \times 10^{-4}$.

FIG. 22. SS wave intensity slope, $g_{\text {exp }}$, vs SS emitted intensity, $I_{0}$, for different temperatures, $\tau$. The values of $\tau$ are written near the curves. The dashed lines are linear fits in logarithmic coordinates: $g_{\exp } \sim I_{0}^{\nu(\tau)}$. The inset: the value of the power index $\nu$ as a function of $\tau$. The $\nu(\tau)$ dependence is linearized in $\tau$-reciprocal coordinates and fitted by the expression: $\nu=\tau_{0} / \tau-\nu_{0}$, where $\tau_{0}=5.2 \times 10^{-4}$ and $\nu_{0}=1$.

FIG. 23. SS wave intensity vs FS amplitude measured by the bolometer located opposite to the heater emitted the SS wave in the first cell for the heater power $w_{h}=108.5 \mu \mathrm{W} / \mathrm{cm}^{2}$, and for $\tau=5.21 \times 10^{-4}$ 
This figure "figs01.gif" is available in "gif" format from: http://arxiv.org/ps/cond-mat/0104414v1 
This figure "fig02.gif" is available in "gif" format from: http://arxiv.org/ps/cond-mat/0104414v1 
This figure "fig03.gif" is available in "gif" format from: http://arxiv.org/ps/cond-mat/0104414v1 
This figure "fig04.gif" is available in "gif" format from: http://arxiv.org/ps/cond-mat/0104414v1 
This figure "fig05.gif" is available in "gif" format from: http://arxiv.org/ps/cond-mat/0104414v1 
This figure "fig06.gif" is available in "gif" format from: http://arxiv.org/ps/cond-mat/0104414v1 
This figure "fig07.gif" is available in "gif" format from: http://arxiv.org/ps/cond-mat/0104414v1 
This figure "fig08.gif" is available in "gif" format from: http://arxiv.org/ps/cond-mat/0104414v1 
This figure "fig09.gif" is available in "gif" format from: http://arxiv.org/ps/cond-mat/0104414v1 
This figure "fig10.gif" is available in "gif" format from: http://arxiv.org/ps/cond-mat/0104414v1 
This figure "fig11.gif" is available in "gif" format from: http://arxiv.org/ps/cond-mat/0104414v1 
This figure "fig12.gif" is available in "gif" format from: http://arxiv.org/ps/cond-mat/0104414v1 
This figure "fig13.gif" is available in "gif" format from: http://arxiv.org/ps/cond-mat/0104414v1 
This figure "fig14.gif" is available in "gif" format from: http://arxiv.org/ps/cond-mat/0104414v1 
This figure "fig15.gif" is available in "gif" format from: http://arxiv.org/ps/cond-mat/0104414v1 
This figure "fig16.gif" is available in "gif" format from: http://arxiv.org/ps/cond-mat/0104414v1 
This figure "fig17.gif" is available in "gif" format from: http://arxiv.org/ps/cond-mat/0104414v1 
This figure "fig18.gif" is available in "gif" format from: http://arxiv.org/ps/cond-mat/0104414v1 
This figure "fig19.gif" is available in "gif" format from: http://arxiv.org/ps/cond-mat/0104414v1 
This figure "fig20.gif" is available in "gif" format from: http://arxiv.org/ps/cond-mat/0104414v1 
This figure "fig21.gif" is available in "gif" format from: http://arxiv.org/ps/cond-mat/0104414v1 
This figure "fig22.gif" is available in "gif" format from: http://arxiv.org/ps/cond-mat/0104414v1 
This figure "fig23.gif" is available in "gif" format from: http://arxiv.org/ps/cond-mat/0104414v1 